Feedback inhibition by GABAergic interneurons that are driven by dorsal raphe-projecting pyramidal neurons of the medial prefrontal cortex

suppress feeding of adolescent female mice undergoing activity-based anorexia

by

Muzi Du ${ }^{1, \#, 5}$, Adrienne Santiago ${ }^{1, \#, 4}$, Cenk Akiz ${ }^{1}$, Chiye Aoki ${ }^{1,2,3}$

${ }^{\#}$ Shared first authors

${ }^{1}$ Center for Neural Science, New York University, New York, NY 10003

${ }^{2}$ Neuroscience Institute, NYU Langone Medical Center, New York, NY, 10016

${ }^{3}$ Corresponding author. Email ca3@nyu.edu, ORCID 0000-0003-4010-9425

${ }^{4}$ Current address of AS: New York State Psychiatric Institute, Columbia University Irving Medical Center, New York, NY, 10032

${ }^{5}$ Current address and ORCID of MD: 0000-0002-5422-6115, The Solomon H. Snyder Department of Neuroscience, Johns Hopkins University, School of Medicine, Baltimore, MD 21205, USA 


\section{Abstract 250}

Anorexia Nervosa (AN) is characterized by voluntary food restriction, excessive exercise and mortality rate surpassing that of major depression. Activity-based anorexia (ABA) is an animal model that captures these characteristics of $\mathrm{AN}$, thus having the potential to reveal the neurobiology underlying individual differences in AN vulnerability. Dorsal raphe (DR) is known to regulate feeding but its role in ABA remains unexplored. Through chemogenetic activation, we investigated the role of $\mathrm{mPFC}$ pyramidal neurons projecting to DR $(\mathrm{mPFC} \rightarrow \mathrm{DR})$ in an animal's decision to eat or exercise following ABA induction. Although the DREADD ligand C21 could activate $44 \%$ of the mPFC $\rightarrow$ DR neurons, this did not generate significant group mean difference in the amount of food intake, compared to control ABA mice without chemogenetic activation. However, further analysis of individual animals' responses to C21 revealed a significant, positive correlation between food intake and $\mathrm{mPFC} \rightarrow \mathrm{DR}$ neurons that co-express cFos, a marker for neuronal activity. cFos expression by GABAergic interneurons (GABA-IN) in $\mathrm{mPFC}$ was significantly greater than that for the control ABA mice, indicating recruitment of GABA-IN by mPFC $\rightarrow$ DR neurons. Electron microscopic immunohistochemistry (EM-ICC) revealed that GABAergic innervation is $60 \%$ greater for the $\mathrm{PFC} \rightarrow \mathrm{DR}$ neurons than the Layer 5 pyramidal neurons without projections to DR. Moreover, individual differences in this innervation correlated negatively with food intake specifically on the day of C21 administration. We propose that C21 activates two antagonistic pathways: 1) PFC $\rightarrow$ DR pyramidal neurons that promote food intake; and 2) GABA-IN in the mPFC that dampen food intake through feedback inhibition of $\mathrm{mPFC} \rightarrow \mathrm{DR}$ neurons.

\section{Key Words:}

anorexia nervosa, serotonin, food restriction, stress-induced anxiety, feedback inhibition 


\section{Declarations:}

Funding: The National Institutes of Health (EY13079; F31 MH112372; R25GM097634); the

National Science Foundation (DBI-1460880); and New York University (NYU Research

Challenge Fund; NYU Dean's Dissertation Fellowship); Vulnerable Brain Project.

Conflicts of interest/competing interests: None of the authors have conflict of interest to disclose. None of the authors have relevant financial or non-financial interests to disclose.

Availability of data: Raw data of wheel counts, body weight, food consumption, and images captured using the confocal and electron microscopes can be provided upon request.

Code availability: not applicable.

Authors' contributions: All authors read and consented to the submitted manuscript. The original text was written by $\mathrm{M}$. Du, then extensively revised by $\mathrm{C}$ Aoki and edited in its final stage by A. Santiago and C. Akiz. A. S performed all of the surgeries. Daily care of the animals was performed by A.S. and C. Aoki. All authors contributed to data analysis. AS and MD performed analysis of wheel running, body weight and food consumption. MD and C Akiz performed analysis of immunofluorescent data. MD and C Aoki performed analysis of EM data.

Ethics approval: All aspects of live animal handling followed the protocol approved by NYU's University Animal Welfare Committee and by the Institutional Animal Care and Use Committees of New York University (A3317-01).

Consent of publication: all authors consent to the publication of the submitted manuscript. 
Acknowledgements: The authors thank the help of the following undergraduates in animal husbandry and daily data collection: Sabrina George, Ishan Handa, Emily Makowicz, Rose Temizer 


\section{Introduction}

Anorexia Nervosa (AN) is an eating disorder that affects approximately 2.1 million people (Collaborators, 2013). The characteristic symptoms of AN include voluntary food restriction in pursuit of thinness, compulsive and over-valued thoughts on body shape and weight, as well as failure in maintaining healthy body weight (Attia, 2010). AN is highly comorbid with anxiety and mood disorders (Hudson, Hiripi, Pope, \& Kessler, 2007; Merikangas et al., 2010), and has a mortality rate of around 10\%. AN is still without approved pharmacological treatment, making AN one of the deadliest among mental illnesses (Arcelus, Mitchell, Wales, \& Nielsen, 2011; Birmingham, Su, Hlynsky, Goldner, \& Gao, 2005). The neurobiological mechanisms and the treatment for AN remain unresolved.

A rodent model called Activity-Based Anorexia (ABA) captures several key symptoms of AN. Upon giving mice free access to running wheels prior to a few days with restricted food access, some but not all animals undergo voluntary hypophagia and increased wheel running (Hall, Smith, Schnitzer, \& Hanford, 1953). These behaviors are maladaptive, since hyperactivity does not improve food access, but instead causes heightened energy expenditure that could be lethal. The major difference between ABA and AN is that, in the ABA model, food restriction (FR) is initially imposed by experimenters. However, this phase is followed by voluntary FR, one hallmark of $\mathrm{AN}$, in that animals choose to run rather than eat, even during the limited hours of food availability. In sum, ABA captures the following essential elements of AN: Severe weight loss, extensive exercise even during the limited hours of food availability, and anxiety. The significance of this model lies in its potential to shed light on the neurobiological underpinnings of anorexia-like behaviors in rodents (Chowdhury et al., 2019; Chowdhury, Wable, Sabaliauskas, \& Aoki, 2013; Foldi, Milton, \& Oldfield, 2017; Santiago, Makowicz, Du, \& Aoki, 2021), through which the mechanism of as well as the treatments for patients with AN can be enlightened. 
Adolescence is the period when individuals are the most susceptible to AN (Kaye, Fudge, \& Paulus, 2009). This period is marked by significant changes in metabolism, endocrine system and brain development, but also by individuals' psychological and sociocultural transitions (Connan, Campbell, Katzman, Lightman, \& Treasure, 2003; Fuhrmann, Knoll, \& Blakemore, 2015; Giedd et al., 1999). Those transitions, whether physiological or mental, could be stressful for individuals. As a result, despite an increased resilience to physical diseases and injuries, the mortality rate during adolescence doubles rather than decreases (Dahl, 2004). Therefore, the intense stress faced during this adolescent transition might contribute to the development of eating disorders such as AN. Indeed, individuals diagnosed with AN commonly report the experience of mental stress (Ball \& Lee, 2000), and AN-like behaviors could be induced by chronic stress in rats (Marti, Marti, \& Armario, 1994). Therefore, the ability to handle stress effectively may be essential for protecting individuals from developing AN.

The neural circuits underlying stress response may be a potential neurobiological target for AN pathology. The dorsal raphe nucleus (DR) is an integral component of the serotonin (5-HT) system in the central nervous system. The DR responds potently to external stress, such as motion restraints and early maternal separation (Grahn et al., 1999; Hardaway, Crowley, Bulik, \& Kash, 2015; Nichols et al., 2017; Pollano, Trujillo, \& Suarez, 2018).Furthermore, a recent study using optogenetic tools revealed a causal relationship between activation of the serotonergic neurons in DR and active coping under inescapable stress in rats and mice (Nishitani et al., 2019). Anatomically, GABAergic interneurons (GABA-IN) in DR receive inputs from Layer 5 pyramidal neurons in the medial prefrontal cortex (mPFC) (Jankowski \& Sesack, 2004). mPFC is implicated in various higher-order cognitive functions such as learning, memory and decision-making and have we have previously implicated this brain region in ABA running (Santiago et al., 2021). Activation of 
$\mathrm{mPFC}$ projections to $\mathrm{DR}(\mathrm{mPFC} \rightarrow \mathrm{DR})$ induces active coping within adverse environments and challenges such as the forced swim test (Warden et al., 2012). mPFC $\rightarrow$ DR also engages the controllability of escapable foot-shock stress, which leads to the blockade of the DRinduced behavioral outcomes in face of inescapable stress (Amat et al., 2005). Those results imply that $\mathrm{mPFC} \rightarrow \mathrm{DR}$ may mediate higher-order control of mental and starvation stress in AN to initiate either active or passive coping strategies such as extensive exercise versus feeding.

Beside its involvement in stress responses, another well-studied role of DR is to regulate feeding behaviors (Bendotti, Garattini, \& Samanin, 1986; Fletcher \& Davies, 1990). DR neurons have differential activities at different phases of feeding, and are significantly more active during ingestion than food searching and satiety (Takase \& Nogueira, 2008). Similarly, while feeding increases the activity of the serotonergic neurons in DR, fasting has the opposite effects of reducing their activities (Nectow et al., 2017). Optogenetic activation of the serotonergic neurons in DR suppresses feeding, while activation of GABAergic interneurons in the same area increases feeding (Nectow et al., 2017). Furthermore, at the molecular level, deletion studies show that the 5-HT4 receptors in the mPFC-DR pathway are required for the development of stress-induced hypophagia (Compan, Walsh, Kaye, \& Geliebter, 2015; Jean et al., 2017). Interestingly, even though feeding involves various sensory modalities such as vision and smell, DR as a feeding regulator actually does not receive any direct input from the primary sensory or motor cortices (Weissbourd et al., 2014). This raises the possibility that the regulation of feeding by DR may involve higher-order controls, such as the voluntary decision-making to restrict food intake seen in individuals diagnosed with AN.

Given the multiple cognitive functions of $\mathrm{mPFC}$, we sought to determine whether $\mathrm{mPFC} \rightarrow \mathrm{DR}$ may affect feeding in the ABA model, in which animals that exhibit anorexia- 
like behaviors make the decision to run or to eat. We report on the feeding behaviors of mice during the ABA schedule in response to the excitation or inhibition of $\mathrm{mPFC} \rightarrow \mathrm{DR}$ pathway. We show strong correlations between the activities of the $\mathrm{mPFC} \rightarrow \mathrm{DR}$ pyramidal neurons in mPFC and the food intake of mice. We further reveal the complex local microcircuits in $\mathrm{mPFC}$ that strongly modulate the $\mathrm{mPFC} \rightarrow \mathrm{DR}$ pathway and feeding of $\mathrm{ABA}$ mice. 


\section{Materials and Methods}

\section{Animals}

Eighteen female mice (wild type C57BL6/J, bred at NYU) were used in this study. Starting from postnatal day 26 (P26), all animals were singly housed at NYU animal facility under a 12:12 light/dark cycle with the light starting at 7 AM and ending at 7 PM. Water and food were provided ad libitum unless when specified. All of the animal handlings adhered to NYU protocols approved by the Institutional Animal Care and Use Committees of New York University (A3317-01).

\section{Surgery for transfection of the mPFC with Multiplexed-Retro DREADD}

In this study, multiplex Designer Receptors Exclusively Activated by Designer Drugs (DREADDs) were used to target the group of neurons projecting from $\mathrm{mPFC}$ to DR. The chemogenetic approach resembled the methods described in another recent publication from this lab, except that the Multiplexed-Retro DREADD induction targeted the mPFC-to-DR, rather than the mPFC pyramidal neurons projecting to dorsal striatum (Santiago et al., 2021). At P26, animals were anesthetized with isoflurane for surgery. For the DREADD experimental animals ( $\mathrm{N}=7$, randomly selected from all of the animals used), viruses containing exogenous genes were transcranially injected through Nanoject II Auto-Nanoliter Injector (\#3-000-204, Drummond) at different sites: DR was injected with a retrogradely transported AAV carrying a Cre-EBFP construct (AAVrg-EBFP-Cre) (Han et al., 2018; Tervo et al., 2016). The volume was 400-500nL, comprised of AAV-rg-pmSyn1-EBFP-Cre (gift from Hongkui Zeng; Addgene viral prep \# 51507-AAVrg; RRID: Addgene_51507; viral titer of $6 \times 10^{12} \mathrm{vg} / \mathrm{mL}$ ). The stereotaxic coordinates for $\mathrm{DR}$ were $\mathrm{AP}=0$ from Lambda, ML +0.8 mm@15, DV 3.3 mm (Correia, Matias, \& Mainen, 2017). The same animals received viruses encoding Cre-dependent hM3D(Gq)-mCherry DREADDs (Gq-DREADDs) (AAV8- 
hSYN-DIO-hM3D(Gq)-mCherry, gift from Bryan Roth, viral prep \# 44361-AAV8; RRID: Addgene_44361; viral titer of $4 \times 10^{12} \mathrm{vg} / \mathrm{mL}$ ) and Cre-dependent KORD-mCitrine DREADDs (KORD) (AAV1-hSYN1-dF-HA-KORD.IRES.mCitrine), a generous gift from Dr Shaham, Yavin of NIDA, produced by the Genetic Engineering and Viral Vector Core of NIDA; viral titer of $2 \times 10^{12} \mathrm{vg} / \mathrm{mL}$ ) simultaneously into $\mathrm{mPFC}$ using the stereotaxic coordinates of AP $1.8 \mathrm{~mm}$ from Bregma, $\mathrm{ML} \pm 0.32 \mathrm{~mm}, \mathrm{DV} 1.5 \mathrm{~mm}$ as a 50:50 mixture, $100 \mathrm{nl}$ per hemisphere. Control group was constructed by injecting viruses encoding the CaMKII $\alpha$-dependent-eGFP (gift from Bryan Roth; Addgene viral prep \#50469-AAV8; RRID: Addgene_50469, $\left.1 \times 10^{13} \mathrm{vg} / \mathrm{mL}\right)$, to express GFP in CaMKII $\alpha+$ pyramidal cells in mPFC without co-expression of DREADD. Alternatively, control animals received the virus encoding Cre-dependent DREADDs but without the retrograde-Cre viruses in DR. The two control subgroups were pooled after verifying through statistical tests that they lacked behavioral differences.

\section{ABA schedule}

The schematic illustrations for the ABA schedule are included in Fig. 1A for the first ABA induction (ABA1) and for the second ABA induction (ABA2, Fig. 2A). 


\section{Figure 1 ABA1 timeline and behavior}

Schematic illustration of the first ABA induction and the daily food restriction (FR) schedule. See Method section for the details of the ABA1 induction. FAA: food anticipatory activity; FA: food availability; PP: post prandial period. (b) individual daily wheel count (WCT); (c) WCT during ABA; (d) Body weight; (e) Food intake during the pre-ABA and the three days of FR. The average daily food intake during the two days before the onset of FR on P41 was used as the Pre-ABA baseline food intake. The average daily food intake during the two days after ABA, on P44, was used as the post-ABA food intake.

a

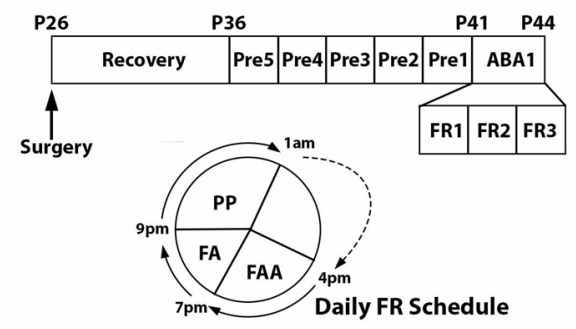

C

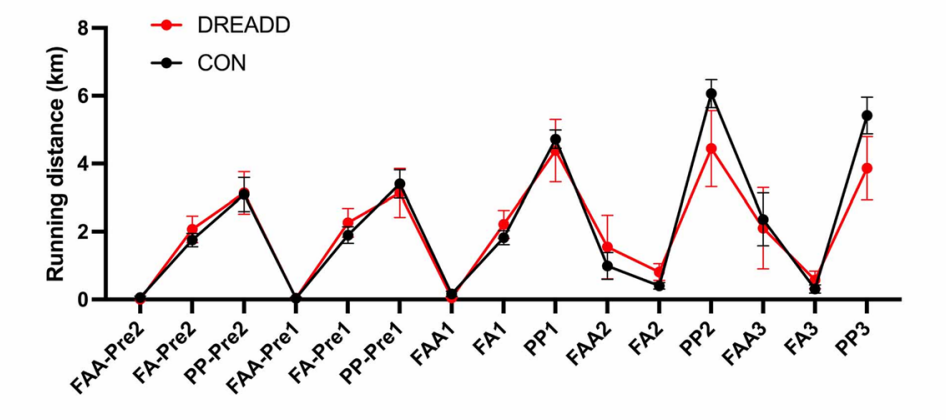

d

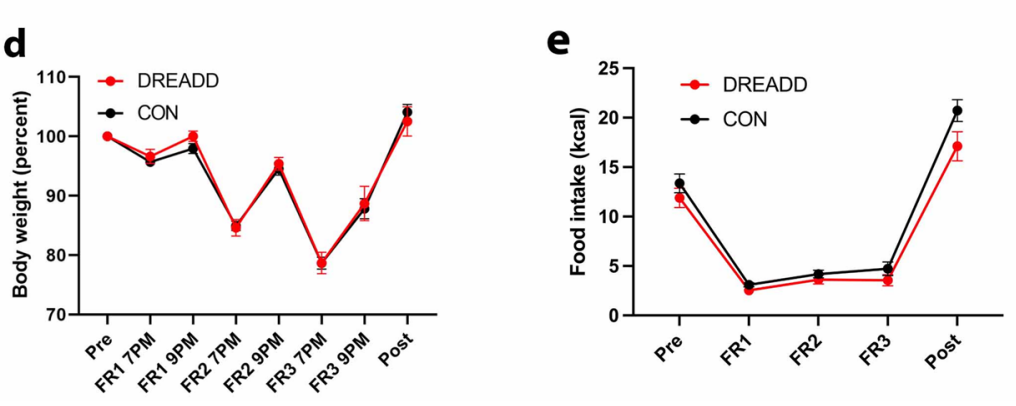

b

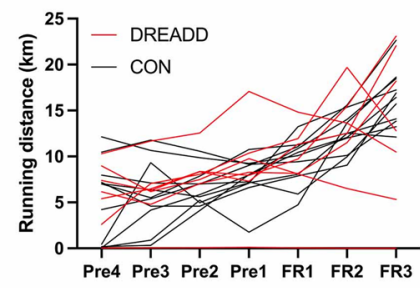




\section{Figure 2 ABA2 timeline and behavior}

Schematic illustration of the second ABA induction. See Method section for the details of the second ABA induction. (b) WCT; (c) body weight; (d) Food intake during the pre-ABA and the four days of food restriction. * indicates $p$ $\leq 0.05$.

a

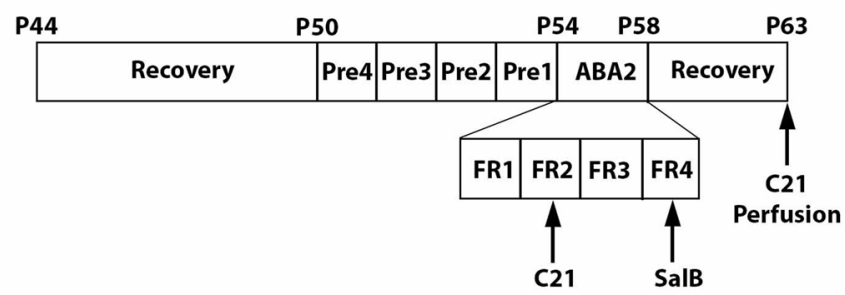

b

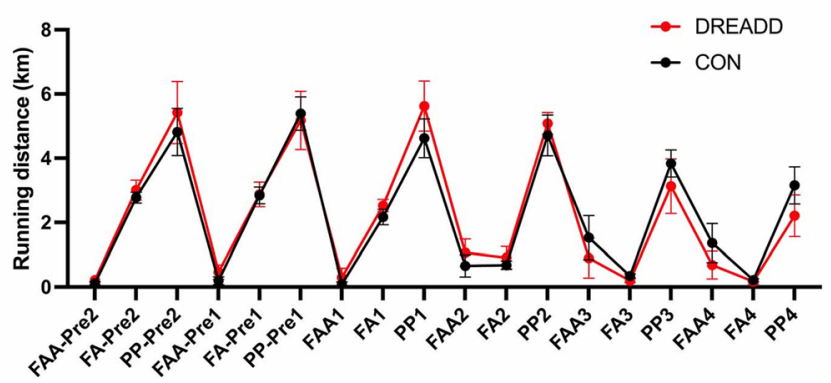

C

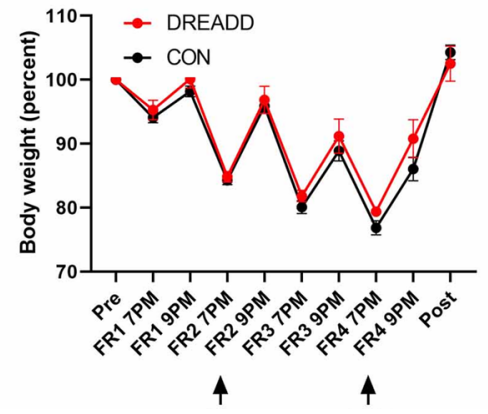

d

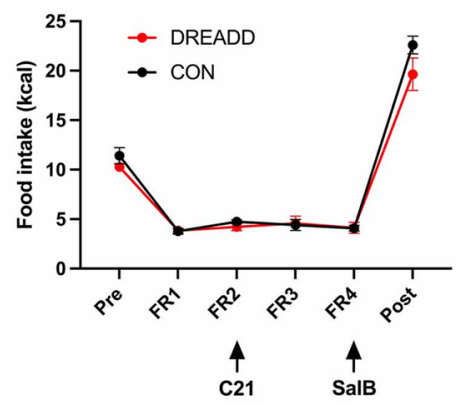


After ten days of recovery from surgery, at P36, an acclimation phase began, whereby animals had 24h/day access to a running wheel (Low-Profile Wireless Running Wheel for Mouse ENV-044, Med Associates, Fairfax, VA) in addition to ad libitum food and water (Pre1, Pre2, Pre3, Pre4, Pre5). The food was of two types: dry food pellet (LabDiet PMI Nutrition Int'1, Brentwood, MO's \#5001, 10\% fat, 20\% protein, 70\% carbohydrate, 4.07 gross energy kca./g, 3.02 metabolizable $\mathrm{kcal} / \mathrm{g})$ and wet food $\left(\mathrm{Clear} \mathrm{H}_{2} \mathrm{O}\right.$ brand DietGel 76A in plastic cups, $0.998 \mathrm{kcal} / \mathrm{g}, 4.7 \%$ protein, $17.9 \%$ carbohydrates, $1.5 \%$ fat, $73.4 \%$ moisture). On P41, the food was removed at 1 PM, leaving the animal only with free access to the running wheel (first ABA induction, ABA1), an empty food hopper and water gels (Clear $\mathrm{H}_{2} \mathrm{O}$ brand Hydrogels Product \#70-01-5022, $0 \mathrm{kcal} / \mathrm{g}$. In the following three days, food access was given only between 7 PM to 9 PM and the animals' food intake during the two-hours of food availability were measured. At noon of P44, ad libitum food access was restored and the running wheel was removed from the cage. After six days of recovery from ABA1, starting on P50, the running wheel was reintroduced to the cage for four days (FR1, FR2, FR3, FR4) followed by a second ABA induction (ABA2) between P54 (1 PM) to P58 (1 PM).

ABA2 was the same as ABA1, with the following exceptions: on P55, GqDREADD's ligand Compound 21 (C21; Tocris, Cat. No. 5548, $1 \mathrm{mg} / \mathrm{kg}$ body weight, suspended in sterile saline from a stock solution dissolved in DMSO) was injected intraperitoneally at 3:30 PM and 9:30 PM; on P57, KORD-DREADD's ligand salvinorin B (SalB; Cayman Chemical, Cat. No. 23582, $10 \mathrm{mg} / \mathrm{kg}$ body weight, dissolved in DMSO, then suspended 1:5 in sunflower oil) was injected subcutaneously at 3:30 PM and 6:30 PM. Starting from 1 PM on P58, the animals went through 7 days of recovery, whereby they had ad libitum access to food and wheel. During one of the days of recovery, C21 was injected, while on another of the days of recovery, SalB was injected,for assessing whether the DREADD ligands' effects on behavior depended on FR. 


\section{Immunocytochemical verification of DREADD gene expression}

Euthanasia was achieved by anesthetizing animals deeply using urethane (1000$1500 \mathrm{mg} / \mathrm{kg}$, I.P.), then transcardially perfusing with the following solutions: $0.1 \mathrm{M}$ phosphate buffer (PB) (pH 7.4) containing 10,000 units/500 ml of Heparin, followed immediately by $4 \%$ paraformaldehyde (PFA) in $0.1 \mathrm{M}$ PB. Brains were extracted from the skull, then post-fixed in 4\% PFA/0.1M PB for at least three days at room temperature. Each brain was blocked and sectioned along the coronal plane at a thickness of $50 \mu \mathrm{m}$ using a vibrating microtome. Brain sections from all DREADD experimental animals $(\mathrm{N}=7)$ were used for the following immunocytochemical processing.

The successful deposition of AAVrg-EBFP-Cre in DR was confirmed using a rabbit anti-GFP primary antibody (Invitrogen (Molecular Probes) Cat \# A11122; RRID AB_221569, 1:2000), taking advantage of its cross-reactivity for detecting EBFP, followed by the use of a goat anti-rabbit IgG for the secondary antibody (Vector Cat \# BA-1000 lot ZA-0924, 1:200) and Vector's Elite kit (PK-6100, Vector Laboratories) with VIP as the color indicator (SK4605, Vector Laboratories) (Fig $3 \mathrm{a}, \mathrm{b}$ ). This immunocytochemical procedure also detected mCitrine, the reporter of KORD (not shown). The expression of Cre-dependent GqDREADD genes was verified using a rat anti-mCherry primary antibody (ThermoFisher, Cat. No. M11217, RRID AB_2536611, 1:1000), a biotinylated goat anti-rat IgG secondary antibody (Vector Laboratories Cat \#BA-9400, lot Z001216, 1:200) and Vector's Elite Kit with VIP as the indicator. A subset of the sections immunolabeled for mCherry were also immunolabeled for cFos, to verify that the pyramidal neurons with immunoreactivity to mCherry were activated by the DREADD ligand, C21. To this end, vibratome sections were co-incubated with rat anti-mCherry and rabbit anti-cFos primary antibody (Cell Signaling Tech, Cat. No. 5348S; RRID AB_10557109, 1:800), followed by co-incubation in 
biotinylated goat anti-rat IgG and biotinylated goat anti-rabbit IgG (Vector Laboratories Cat\# BA-1000, lot ZA-0924, 1:200) secondary antibodies, and visualized using the same kit as above.

Vibratome sections were first incubated for 30 min with $1 \% \mathrm{H}_{2} \mathrm{O}_{2}$ in $0.01 \mathrm{M}$ phosphate buffer $/ 0.9 \%$ sodium chloride (PBS), rinsed in PBS, then were blocked for $30 \mathrm{~min}$ in PBS buffer containing $1 \%$ bovine serum albumin (BSA) and $0.05 \%$ sodium azide. Incubations with the primary antibodies, anti-GFP and anti-mCherry occurred over a period of 1 to 3 days, under constant agitation at room temperature. Following three rinses in PBS, sections were incubated with the secondary antibodies for 1 hour at room temperature, under constant agitations. After this step, sections were processed for the ABC-peroxidase reaction by following the Vector Laboratories' User Guide. Sections were mounted on glass slides, coverslipped and visualized using the Echo Revolve light microscope.

\section{Immunocytochemistry for triple immunofluorescent confocal microscopy}

In order to confirm that $\mathrm{C} 21$ activated Gq-DREADD-transfected pyramidal neurons in the $\mathrm{mPFC}$, and to ascertain whether the firing of pyramidal neurons increased the firing of GABAergic interneurons (GABA-INs) in the mPFC, animals were injected on P63 with C21 approximately 90 minutes prior to euthanasia.

Intact sections that had clear cingulate cortex area, prelimbic cortex and infralimbic cortex ( Bregma $1.98 \mathrm{~mm}$ to $2.32 \mathrm{~mm}$ ) of these experimental animals, together with vibratome sections prepared as described above for the control animals without DREADD expression $(\mathrm{N}=10)$ underwent the immunocytochemical procedure. After incubating sections for 30 min with $1 \% \mathrm{H}_{2} \mathrm{O}_{2}$ in PBS, sections were rinsed in PBS, then were blocked for 30 min in PBS buffer containing $1 \%$ BSA and $0.05 \%$ sodium azide. Sections were then incubated in PBS-BSA-azide containing three primary antibodies: rabbit anti-cFos antibody (Cell 
Signaling Tech, Cat. No. 5348S; RRID AB_2536611, 1:800), rat anti-mCherry antibody (ThermoFisher, Cat. No. M11217, RRID AB_2536611; 1:1000), and mouse anti-glutamic acid decarboxylase 67 (GAD) antibody (Millipore Sigma, Cat. No. MAB5406, clone 1G10.2; RRID AB_2278725, 1:800). After one to three days of incubation at room temperature, brain sections were washed three times with PBS for ten minutes each. Sections then were incubated in PBS-BSA-azide buffer containing the following secondary antibodies: goat anti-rat IgG antibody conjugated to Alexa Fluor 594 (Jackson ImmunoResearch, Cat. No. 112-585-143; $1: 100)$ to recognize anti-mCherry, Affini-pure goat anti-rabbit IgG antibody conjugated to Alexa Fluor 647 (Jackson ImmunoResearch, Cat. No. 111-605-144; 1:100) to recognize anti-cFos, and goat anti-mouse IgG antibody conjugated to DyLight 405 (Jackson ImmunoResearch, Cat. No. 111-475-166,1:100) to recognize anti-GAD. After an overnight incubation in secondary antibodies at room temperature, brain sections were washed three times with PBS for 10 minutes each.

\section{Controls for immunofluorescence microscopy}

We routinely conducted two types of controls. One was to stain sections singly, to ensure no 'bleeding' of fluorescent labels beyond the channel designated for the immunolabel. The second was to pair a primary antibody with a secondary antibody of an inappropriate species, to ensure absence of inter-species cross-reactivity.

\section{Confocal microscopic imaging and analysis}

The brain sections were observed under the confocal microscope Leica Confocal SP8 with Leica's Application Suite 2.6.0 using three channels to visualize three kinds of secondary antibodies separately. Multiple images at a single plane of focus were taken from the mPFC. ImageJ (version 1.52K; RRID: SCR_003070, National Institute of Health, USA) 
was used to analyze the images taken from the three channels of the confocal microscope. For each image, a random window containing cingulate cortex area 1, prelimbic cortex and/or infralimbic cortex was selected and every neuron excluding those lying on the bottom or the right border of this window was counted and their distance to the pia surface was measured. Every neuron with florescence detected by the mCherry channel was treated as an mCherry+ (i.e., Gq-DREADD expressing) neuron. Only a substantial ring-shaped labeling detected by the GAD channel was categorized as GAD+ (i.e., GABA-IN) For the cFos channel, optical density of the nucleus of each labeled neuron was measured. From the histogram, it was determined that cFos intensity greater than 25 or the ratio of cFos intensity divided by their mean value in a single window greater than 0.5664 was regarded to be above the threshold of cFos + (i.e. activated) neuron.

\section{Immunohistochemistry and tissue processing for electron microscopy}

Brain sections from the experimental group $(\mathrm{N}=7)$ were gained in the same way as discussed above for confocal microscopy. Each section underwent freeze-thawing (Wouterlood \& Jorritsma-Byham, 1993) to increase the permeability of cell membranes with minimal disturbance of membranous structures. The immunohistochemistry procedure for dual labeling of GAD and mCherry began by blocking nonspecific immunolabeling by incubating for $30 \mathrm{~min}$ in $0.01 \mathrm{M}$ PBS $/ 0.05 \%$ azide/ $1 \%$ BSA. This was followed by a three days' primary antibody incubation in rat anti-mCherry antibody (ThermoFisher, Cat. No. M11217; RRID AB_2536611, 1:1000) and mouse anti-GAD antibody (MilliporeSigma, Cat. No. MAB5406; RRID AB_2278725, 1:800) dissolved in $\mathrm{pH} 7.6 \mathrm{PBS} / 1 \%$ BSA/0.05\% sodium azide, and an overnight secondary antibody incubation in ultrasmall colloidal goldconjugated goat anti-rat antibody (1:100, EMSciences Hatfield, PA Cat 25181) and biotinylated goat anti-mouse antibody (1:200, Vector Labs BA-1000). After that, sections 
underwent 30 minutes of incubation in avidin-biotinylated horseradish peroxidase complex formation (ABC, Vector's ABC Elite kit), 15 minutes of postfixation using 2\% glutaraldehyde, then silver intensification (KPL Silver Enhancer Kit, Cat \# 5520-0021 to enlarge the ultrasmall colloidal gold particles conjugated to anti-rat IgG, and 3,3'diaminobenzidine (DAB, Sigma Chem Cat No. 5905) substrate reaction. The EM tissue processing was similar to the procedure performed before (Chen et al. 2016), which consisted of post-fixation using $0.1 \%$ osmium tetroxide/ $0.1 \mathrm{M}$ phosphate buffer processing for $30 \mathrm{~min}$, followed by osmium-free tissue processing in $1 \%$ tannic acid, $1 \%$ uranyl acetate and $0.2 \%$ iridium tetrabromide all dissolved in maleate buffer $(\mathrm{pH} 6.0,0.1 \mathrm{M})$, post-fixation in $1 \%$ uranyl acetate in 70\% ethanol (EtOH) overnight, dehydration in 70\%/90\%/100\% EtOH and 100\% acetone, infiltration in EPON 812 (EM Sciences), flat-embed between Aclar plastic sheets (EM Science) and capsule-embedded in BEEM capsules (EM Science). Ultrathin sections were prepared using the Ultracut E ultratomicrotome and collected on nickel grids (EM Science). Lead citrate counterstaining was omitted, so as to facilitate detection of HRPDAB reaction products.

\section{Electron microscopic imaging and analysis}

The imaging window under the electron microscope (EM) was confined to Layer 5 of mPFC (PL, IL and ACA) and each window was required to contain at least two cell bodies with silver intensified gold particles embedded on the plasma membrane and/or in the cytoplasm (mCherry+ neurons). The GABAergic terminals were distinguished from the others based on their HRP/DAB reaction produce appearing more electron-dense than mitochondria and postsynaptic densities in the immediate vicinity. For each animal, approximately $10 \mathrm{mCherry}+$ and 10 mCherry-negative neurons were sampled and quantified. GABAergic terminals that were aligned in parallel with the plasma membrane of the cell 
body were identified to be forming inhibitory synapses and measured of their lengths using the ImageJ's segmented lengths tool. The lengths spanning the plasma membrane to adjacent GABAergic terminals were also measured using the same ImageJ software.

\section{Statistical analyses}

Statistical analyses were performed using GraphPad Prism versions 8 or 9.2.0 Normality tests were followed by t-tests, one-way ANOVA or nonparametric tests, and simple linear regressions of correlation. 


\section{Results}

\section{Wheel running, food intake and body weight during ABA1}

9 out of 18 animals increased their daily 24-hr wheel running progressively during the acclimation phase of ABA1, when animals had access to a wheel without food restriction (FR) (Pre3 to Pre1, Fig $1 \mathrm{a}, \mathrm{b}$ ). Following commencement of FR on FR1, 13 out of 18 animals increased their wheel running during the hours of 4 PM to 7 PM that preceded the hours of food availability (FA) of 7 PM to 9 PM. This increase during the hours of 1 PM to 7 PM, often referred to in the literature as the food-anticipatory activity (FAA) (Hall et al., 1953) was significantly different during ABA1, relative to values during the acclimation days, Pre1 and Pre2 ( $p=0.0237$, paired t test, Fig. $1 \mathrm{c})$. All animals survived three days of FR, in spite of having lost $23 \%$, on average, of their body weight at the end of ABA1, calculated as follows: Body weight on FR1, FR2 and FR3, normalized to the pre-FR body weight at 1 PM. (Fig. 1 d). This was due to the reduction of daily food intake by $70 \%$, calculated as the difference in the averaged value of food intake during two pre-FR days relative to the averaged value of food intake during three FR days, normalized to the averaged value of food intake during two pre-FR days) (shown as changes in kcal consumed in Fig. 1 e). Within 24 hrs of having returned to ad libitum food availability, their body weights were restored to preFR values (Fig. 1 d).

\section{Wheel running, food intake and body weight during ABA2}

Following ABA1, all animals were returned to an environment with ad libitum food availability and no wheel (P44 to P50). At the end of the recovery phase, at P50, a wheel was returned to each animal's cage to allow for re-acclimation (Pre4 to Pre1). At the end of the reacclimation phase of ABA2, all of the animals resumed or surpassed the level of wheel 
running observed by the end of the acclimation phase of ABA1 (Fig. $2 \mathrm{a}, \mathrm{b}$ ). Following commencement of food restriction on FR1 of ABA2, 5 out of 18 animals increased their wheel running during the hours preceding the feeding hours of 7 PM to 9 PM. By FR2, this increase reached significance, relative to the average running during the hours of 4 PM to 7 PM on Pre2 and Pre1_days that preceded FR ( $p=0.04577$, paired t test, Fig. 2 b). All animals survived four days of FR, in spite of having reduced food intake by $60 \%$ (expressed in kcal food intake in Fig. 2 d) and lost $21 \%$ of their body weight (Fig. 2 c). Within 24 hrs, their body weights were restored to pre-FR values (Fig. 2 c).

\section{The effect of C21/Gq-DREADD activation and SalB/KORD-DREADD inhibition of mPFC $\rightarrow$ DR neurons on wheel running}

At 3:30 PM and 9:30 PM on FR2 of ABA2, all animals of the cohort received 1 $\mathrm{mg} / \mathrm{kg}$ of C21 by intraperitoneal injections (Fig. 2 a). Comparisons of wheel running of animals transfected with Gq-DREADD in mPFC versus the controls without Gq-DREADD transfections revealed no group difference on FR2 during the 3 hours preceding feeding (FAA, 4 PM-7 PM, Mann-Whitney test, $\mathrm{p}=0.4240$ ), during the 2 hrs of food availability (FA, 7 PM-9 PM, Welch's t test, $\mathrm{p}=0.5413$ ), or postprandially (PP, 9 PM-1 AM, Welch's t test, $\mathrm{p}=0.6170$ ) (Fig. 2 b). The average wheel running during the 2 hrs post-injection of C21 (4 PM-6 PM) also did not reveal any group difference (Mann-Whitney test, $\mathrm{p}=0.3247$ ).

At 3:30 PM and 6:30 PM on FR4 of ABA2, all animals of the cohort received 10 $\mathrm{mg} / \mathrm{kg}$ of SalB by intraperitoneal injections to suppress activity of the $\mathrm{mPFC} \rightarrow \mathrm{DR}$ pyramidal neurons (Fig. 2 a). No significant difference in wheel running on FR4 was found during 3 hours preceding feeding (FAA, 4 PM-7 PM, Mann-Whitney test, $\mathrm{p}=0.1854$ ), during the 2 hours following the first injection of the DREADD ligand (4 PM - 6 PM, Mann-Whitney 
test, $\mathrm{p}=0.6907$ ), during the 2-hrs of food availability (FA, Welch's t test, $\mathrm{p}=0.6403$ ) or postprandially (9 PM-1 AM, Welch's t test, p=0.2942) (Fig. 2 b).

\section{The effect of C21/Gq-DREADD activation and SalB/KORD-DREADD inhibition of mPFC $\rightarrow$ DR pyramidal neurons on food consumption}

Previous studies have uncovered that $\mathrm{mPFC} \rightarrow \mathrm{DR}$ pyramidal neurons project mostly to the GABA-INs in DR (Jankowski \& Sesack, 2004), and that direct activation of those inhibitory neurons in DR promotes food intake (Nectow et al., 2017). Thus, we predicted that $\mathrm{C} 21 / \mathrm{Gq}-\mathrm{DREADD}$ activation of $\mathrm{mPFC} \rightarrow \mathrm{DR}$ neurons would promote feeding during the limited hours of food availability on FR2 of ABA2. Contrary to this expectation, activation of $\mathrm{mPFC} \rightarrow \mathrm{DR}$ pyramidal neurons yielded no significant difference in the group mean value of feeding by the DREADD group, compared to the control ABA animals without activation of DREADDs (Welch's t test, p=0.2352) (Fig. 2 d). SalB/KORD-DREADD inhibition of $\mathrm{mPFC} \rightarrow \mathrm{DR}$ neurons on FR4 of ABA2 also did not yield significant differences in the group mean value of food intake across the DREADD versus control groups (Mann-Whitney test, $\mathrm{p}=0.4651)($ Fig. $2 \mathrm{~d})$.

\section{Rationale for the anatomical experiments}

Although chemogenetic modulation of the $\mathrm{mPFC} \rightarrow \mathrm{DR}$ pyramidal neurons did not yield statistically significant group mean differences in wheel running or food consumption, relative to controls lacking the expression of DREADDs, we noted substantial individual differences in these behaviors. To verify that DREADD modulated neuronal activity and to assess whether individual differences in behavior might be related to individual differences in the microcircuitry of the mPFC activated by $\mathrm{C} 21$, we first confirmed that DREADD genes 
were expressed in mPFC pyramidal neurons, including their axon terminals of DR, then quantified the proportion of $\mathrm{C} 21$-activatable mPFC neurons by immunofluorescence.

\section{Confirmation of DREADD expression in mPFC pyramidal neurons and DR axons by immunocytochemistry}

Immunocytochemistry was performed to detect EBFP, the reporter protein of the retrogradely transported AAV expressing the Cre protein (AAVrg-EBFP-Cre, Fig 3 a and b). As expected, EBFP was expressed at high density within neurons located in DR, where the virus was injected stereotaxically (Fig 3 b). The Cre-dependent expression of DREADD genes in the perikaryal cytoplasm and dendrites of mPFC pyramidal neurons was verified by the immunoreactivity of these cells to the reporter protein, mCitrine, of KORD (not shown) and of the reporter protein, mCherry of Gq-DREADD (Fig $3 \mathrm{c}$ and f). High density of axon terminals with immunoreactivity to mCherry were evident in DR (Fig 3 d) as were axons with immunoreactivity to mCitrine (not shown). As expected (Anastasiades \& Carter, 2021), a much lower density of fine-caliber, varicose axon terminals, presumably axon collaterals of mPFC pyramidal neurons projecting to the pyramidal tract, were detected within the medial subregion (i.e., near the lateral ventricles) of dorsal striatum, among immunoreactive fiber bundles of the internal capsule (Fig 3 e). 
Figure 3 Confirmation of DREADD expression in the $\mathrm{mPFC} \rightarrow \mathrm{DR}$ neurons and representative triple IF confocal images of mPFC neurons

(a) Schematic of the stereotaxic injection of AAVrg-EBFP-Cre into DR and of the cre-dependent DREADD-AAV into mPFC.

(b) Verification of AAVrg-EBFP-Cre deposition into DR. Note that the region with the highest density of EBFP-immunoreactivity is in the DR. ca=cerebral aqueduct; $\mathrm{PAG}=$ periaqueductal grey; $\mathrm{mlf}=$ medial longitudinal fasciculus. $\mathrm{Bar}=500 \mu \mathrm{m}$.

(c) Verification of Gq-DREADD-mCherry expression in Layer 5 pyramidal neurons of the mPFC. mCherry-immunoreactivity is prevalent within cell bodies in Layer 5, apical dendrites traversing across Layers $2 / 3$ and branching in Layer 1 . The section was dually labeled using anti-cFos, resulting in immunoreactivity of nuclei of the same population of pyramidal neurons. Bar $=100 \mu \mathrm{m}$.

d) Verification of Gq-DREADD-mCherry expression in puncta of DR. Vibratome sections containing DR were processed in parallel with sections containing $\mathrm{mPFC}$ that is shown in panel c. Puncta reflecting mCherry-immunoreactive axon terminals are evident in DR, ventral (filled arrowheads) and lateral to the cerebral aqueduct. Nuclei immunoreactive for cFos are also evident (open arrows) in DR. (e) Verification of Gq-DREADD-mCherry expression in axons coursing within fiber bundles of the internal capsule (open arrowheads) and varicose axonal processes (filled arrowheads) in dorsal striatum. Calibration bar $=50 \mu \mathrm{m}$ and applies to panels $\mathrm{d}$ and $\mathrm{e}$.

(f) Overview of the section showing mCherry (green), cFos (red) and GAD (blue) immunofluorescence. Note that most of the mCherry signals were contained in the prelimbic (PL) and infralimbic (ILA) areas, which constitute major parts of mPFC. ACA, anterior cingulate area; fa, anterior forceps. Scale bar $=170 \mu \mathrm{m}$. (g) Laminar distribution of mCherry, cFos and GAD immunolabeling. Layers were identified according to Allen Brain Atlas based on the distance to pia: Layer 1, 0-125 $\mu \mathrm{m}$; Layers 2/3, 125-325 $\mu \mathrm{m}$; Layer 5, 325-525 $\mu \mathrm{m}$; Layer 6, >525 $\mu \mathrm{m}$. Scale bar $=50$ $\mu \mathrm{m}$; (h) Representative image of an $\mathrm{mCherry}+/ \mathrm{cFos}+$ neuron (filled arrowhead), mCherry $+/ \mathrm{cFos}-$ negative neurons (open arrowheads), and an mCherry-negative/cFos + neuron (arrow). Scale bar $=25$ $\mu \mathrm{m}$; (i) Representative image of a GAD $+/ \mathrm{cFos}+$ neuron (filled arrowhead), a GAD $+/ \mathrm{cFos}-$ negative neuron (open arrowhead), and an mCherry + dendrite with spines (asterisk). Scale bar $=10 \mu \mathrm{m}$. 


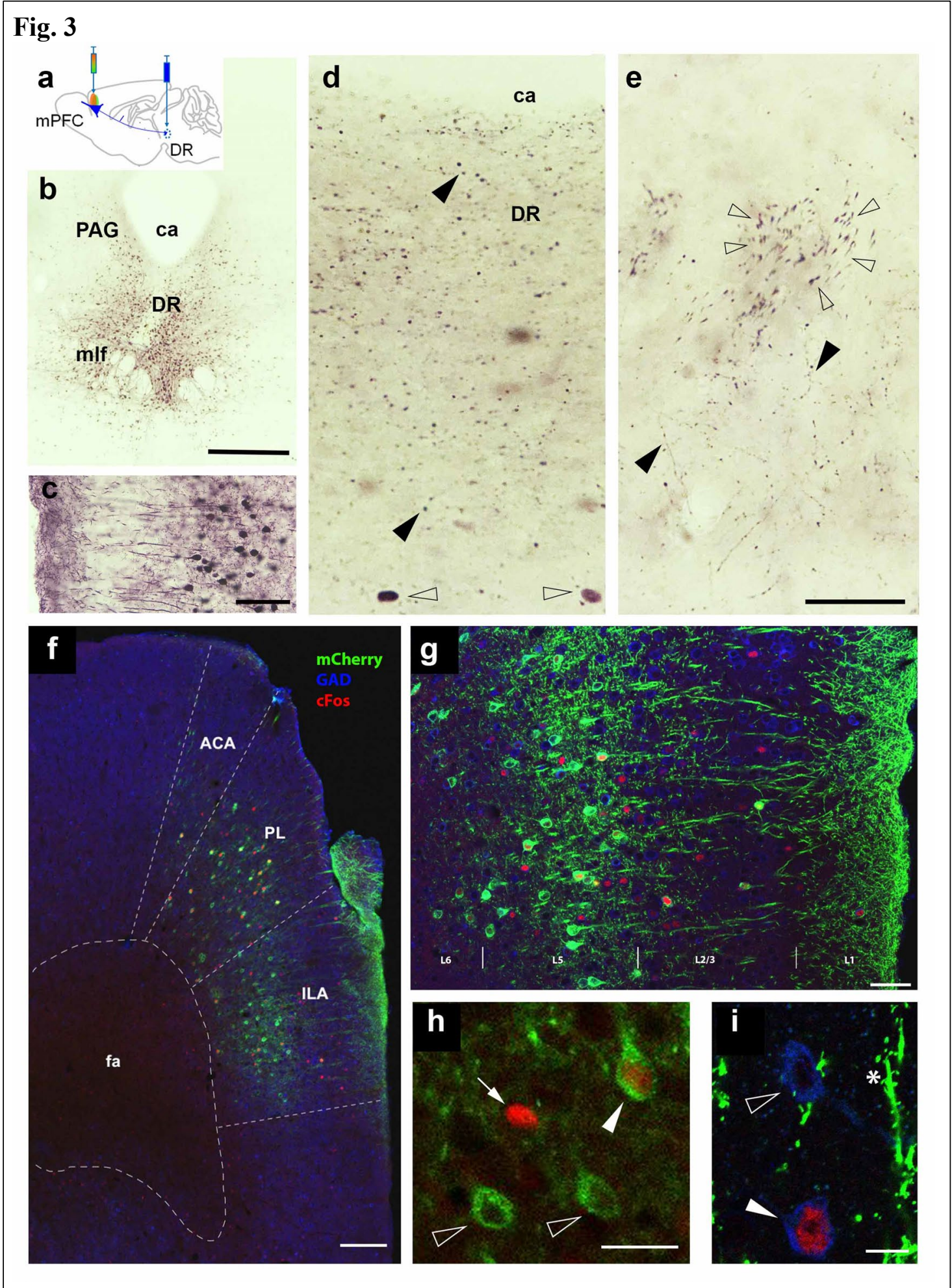




\section{Validation of Gq-DREADD expression in Layer 5 of $\mathrm{mPFC}$ within tissue immunolabeled triply for mCherry, cFos and GAD}

Brain sections containing $\mathrm{mPFC}$ were examined under the confocal microscope after immunohistochemical processing. Representative images are shown in panels f through i of Fig 3. The expression of mCherry-tagged Gq-DREADDs in perikaryal cytoplasm was localized to Layer 5 and mainly confined in the prelimbic and infralimbic areas (Fig. 3 f). This laminar pattern in cortex was as expected, based on the known laminar distribution of mPFC pyramidal neurons with projections to DR (Jankowski \& Sesack, 2004) as well as the targeted locations of stereotactically injected rgAAV-EBFP-Cre virus in DR and of the Credependent AAV-DREADD in cerebral cortex. At a higher magnification, the mCherry-tagged Gq-DREADD-expressing cells were verified to be pyramidal neurons based on their long singular apical dendrites spanning through Layer $2 / 3$ and 1 of mPFC (Fig. 3 g), as well as the prevalence of dendritic spines (white asterisk, Fig. 3 i).

Besides mCherry, sections were co-labeled for the immediate early gene product, cFos, an indicator of neuronal firing, and glutamic acid decarboxylase (GAD), a synthetic enzyme for GABA and thus an indicator of GABAergic inhibitory neurons. As shown in Figure $3 \mathrm{~g}$ and $\mathrm{h}$, cFos immunoreactivity was restricted to the nucleus, which conforms to the expression and the nuclear translocation of cFos protein upon neuronal firing (Bullitt, 1990; Roux et al., 1990). GABAergic interneurons (GABA-IN) were identified by GADimmunoreactivity exclusively within the cytoplasm and their relatively small cell body diameter (Fig. 3 d).

\section{Laminar distributions of mCherry + , cFos + and GAD+ neurons}

The laminar distributions of mCherry + , cFos + and GAD + neurons (Fig. 4 a) were assessed quantitatively and separately by calculating the relative fraction of the indicated cell 
type within $50 \mu \mathrm{m}$ bins centered at a position specified by its distance to the pial surface. As

shown in Fig. 4 b, $\sim 80 \%$ of the total mCherry+ neurons were located within $325-525 \mu \mathrm{m}$

from pial surface, corresponding to Layer 5, as was expected (Jankowski \& Sesack, 2004).

Fig 4 Laminar distributions of mCherry+, GAD+ and cFos+ neurons

(a) The same window within ILA from a DREADD experimental animal captured by GAD, mCherry and cFos immunofluorescence channels, respectively. The pial surface is located at the very bottom of the image. Scale bars $=50 \mu \mathrm{m}$. (b) The fraction of mCherry + and cFos + neurons distributed along laminae for the DREADD experimental group $(\mathrm{N}=7)$ and the control group $\left(\mathrm{N}^{\prime}=10\right)$. (c) The fraction of GAD+ neurons distributed along the laminae for the DREADD experimental group $(\mathrm{N}=7)$ and the control group $\left(\mathrm{N}^{\prime}=4\right)$. Each data point represents the relative fraction of the indicated cell type within a $50 \mu \mathrm{m}$ bin centered at the position specified by its $\mathrm{X}-$ coordinate. Data are shown as mean \pm SEM.

a

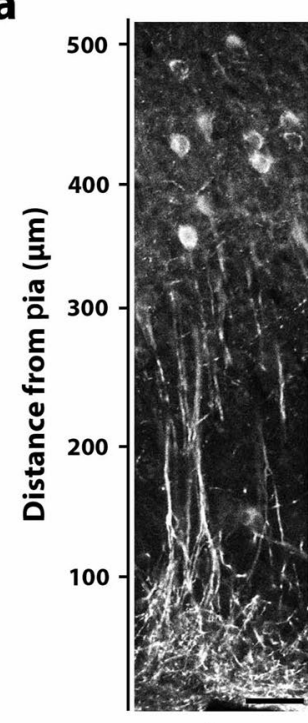

mCherry

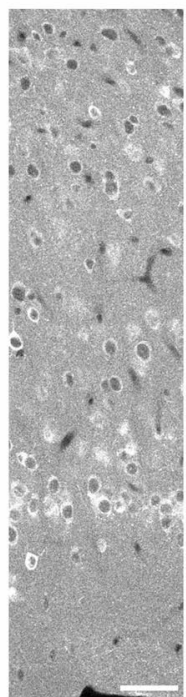

GAD

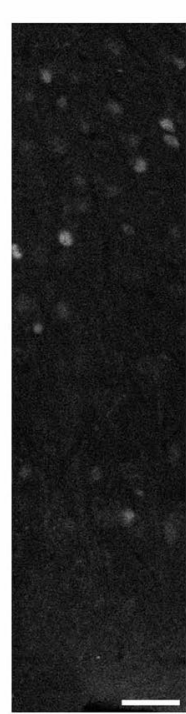

cFos b
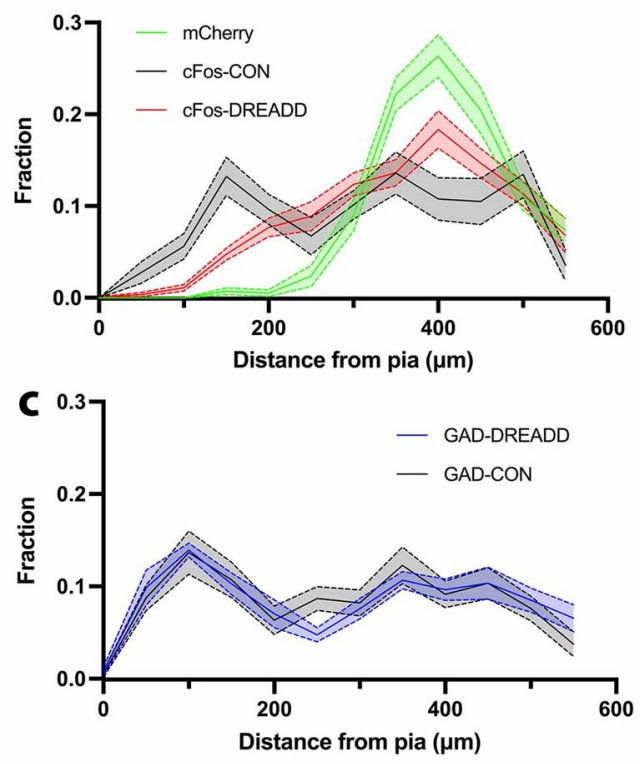
The cFos laminar distributions were assessed for both the DREADD experimental animals and the control animals without DREADD expression (Fig. 4 b). For the DREADD experimental group, most of the cFos + neurons were located around $400 \mu \mathrm{m}$ to pia, which coincided with the pinnacle of the distribution of mCherry+ neurons (Fig. 4 b). For the control group, however, cFos distribution showed a smaller fraction at around $400 \mu \mathrm{m}$ from pia but a relatively greater fraction around Layer 2/3 (Fig. 4 b). This indicates that GqDREADDs effectively drove neuronal firing in Layer 5, where most of the DREADDs were expressed. Interestingly, while less than $13 \%$ of the mCherry+ neurons were distributed at around $125-325 \mu \mathrm{m}$, corresponding to Layers $2 / 3$, the fraction of $\mathrm{cFos}+$ neurons within the same layer of the experimental animals accounted for as much as $\sim 34 \%$ of the total cFos population (Fig. 4 b). This indicates that neurons in Layers $2 / 3$ were also activated, although unlikely by direct Gq-DREADD modulation.

As for the GAD+ neurons, their laminar distributions in the experimental and the control groups were similar (Fig. 4 c): both peaked within Layer 1 and were relatively evenly distributed across the deeper layers.

\section{Density of cFos+ neurons is increased by C21/G1-DREADDs in Layers 5 and 2/3}

For both the experimental and the control groups, the cFos + neuronal density in $\mathrm{mPFC}$ was calculated within $50 \mu \mathrm{m}$-wide windows along the laminae of $\mathrm{mPFC}$ and centered at a position specified by their distance to pial surface, as described above for the laminar distribution of the three cell types (Fig. 5 a). The value of cFos + neuronal densities across at varying distances from pia were averaged across $\sim 4$ confocal microscope windows for each animal. Across laminar distances ranging from 200-550 $\mu \mathrm{m}$ from pia (Layer 2 through the start of Layer 6), the cFos+ neuronal density was significantly greater for the DREADD experimental group than the control group, (Fig. 5 b). The cFos + densities in Layers $2 / 3$ and 
5 were both significantly greater for the DREADD animals than controls (Fig 5 c, Mann-

Whitney $U=6.000, P=0.003085$ for Layer 2/3; Mann-Whitney $U=3.000, P=0.000720$ for

Layer 5). These results further reveal that C21/Gq-DREADDs elevated the activities not only of Layer 5 neurons but also of Layer 2/3 neurons.

\section{Figure 5 cFos + neuron density across the laminae}

(a) Representative confocal images showing the $\mathrm{cFos}+$ neurons in PL along the laminae of a DREADD experimental animal and a control animal. The pia surface is located at the very bottom of the image. Scale bars $=50 \mu \mathrm{m}$. (b) The density of cFos + neurons across the laminae. Each data point was represented by the density of cFos + neurons in a $50 \mu \mathrm{m}$ high window centered at the position specified by its $\mathrm{x}$-coordinate. The width of the windows varied from image to image, but was typically around 300-400 $\mu \mathrm{m}$, covering PL and/or ILA. Data are shown as mean \pm SEM. (c) The density of $\mathrm{cFos}+$ neurons by layers. Layer categorization was as described in Figure $3 . * \mathrm{p} \leq 0.05$, ** $\mathrm{p} \leq 0.01, * * * \mathrm{p} \leq 0.001, * * * * \mathrm{p} \leq 0.0001$ by the Mann-Whitney test.

a

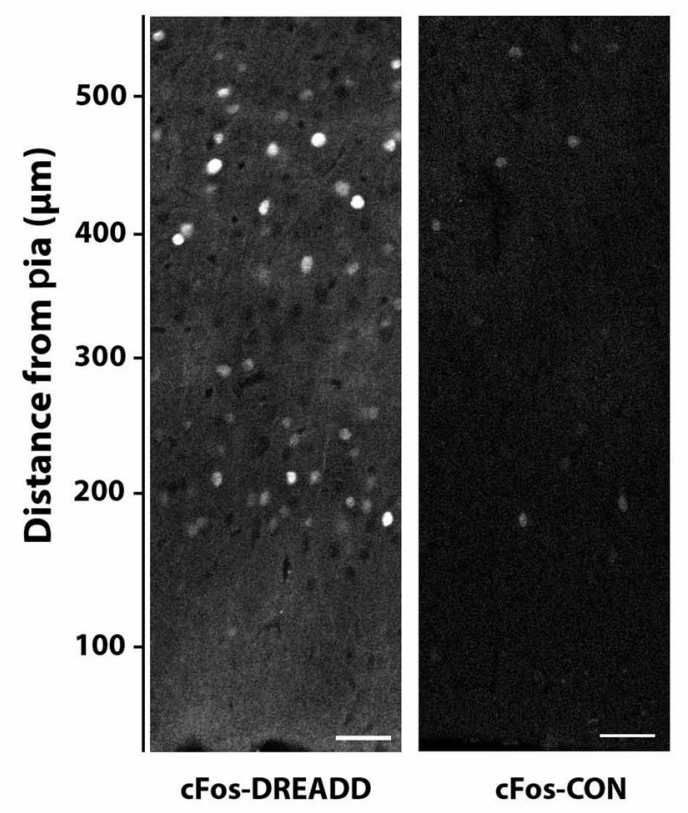

b

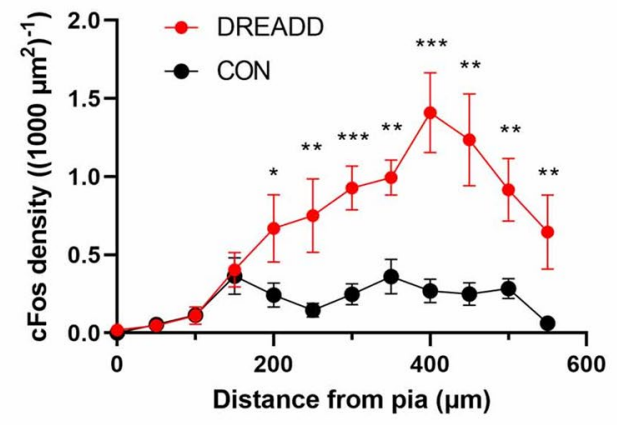

C

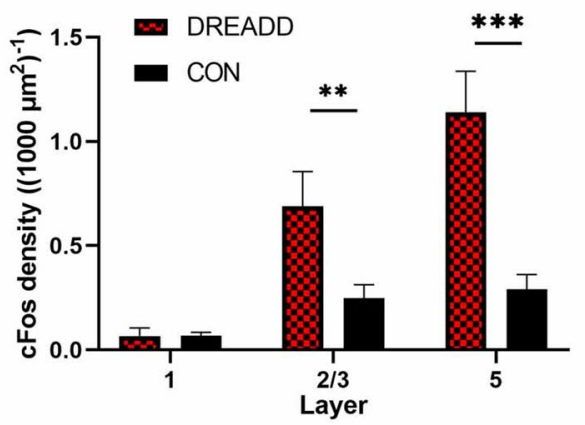




\section{Activities of GABAergic neurons}

The cFos + density quantification described above did not differentiate GABAergic versus pyramidal neurons. The activities of the GABAergic subset of neurons in $\mathrm{mPFC}$ were examined through immunofluorescence as well. For each animal, the averaged percentage of $\mathrm{GAD}+$ neurons that co-expressed cFos $(\% \mathrm{GAD}+/ \mathrm{cFos}+$, Fig. $3 \mathrm{~d})$ captured from all windows that covered mPFC layers was calculated. The $\% \mathrm{GAD}+/ \mathrm{cFos}+$ for the DREADD experimental group was significantly greater than that for the control group (Fig. 6 a; Welch's $\left.t_{(7.407)}=2.937, P=0.0205\right)$. Notably, in the mPFC of DREADD animals, $\% \mathrm{GAD}+/ \mathrm{cFos}+$ showed a positive correlation with the percentage of mCherry + neurons that co-expressed cFos (\%mCherry $+/ \mathrm{cFos}^{+}$, Fig. 6 b). This correlation was nearly significant $\left(r^{2}=0.5507, P=0.0562\right)$.

Laminar analysis of the cFos $+/ \mathrm{GAD}+$ cells revealed that Gq-DREADD activation increased the firing of GABA-INs across Layers $2 / 3$ and 5 of the DREADD animals more than of CONs (Fig. 6 c; $p=0.0203$ and Mann-Whitney $U=12.00$ for Layers $2 / 3 ; p=0.010$ and Mann-Whitney $U=9.5$ for Layer 5).

The great majority of the $\mathrm{cFos}+/ \mathrm{GAD}$-negative cells were presumed to be pyramidal. This category of neurons in Layers 2/3 and 5 were also of higher density for the DREADD animals than for the CON animals (Fig. 6 c; $p=0.0068$ and Mann-Whitney $U=8.000$ for Layers 2/3; $\mathrm{p}=0.0012$ and Mann-Whitney $\mathrm{U}=4.00$ for Layer 5). Moreover, among the cFos + cells, those that were both GAD-negative and mCherry-negative in Layer were also more prevalent in the mPFC of DREADD animals than of the CON animals ( $p=0.0031$, MannWhitney $U=6.00$ ). These were presumed to include the pyramidal neurons without projections to DR. 
Overall, these observations indicate that GABA-INs, pyramidal neurons of Layers

$2 / 3$, and pyramidal neurons in Layer 5 without projections to DR were all driven to fire more, via the Gq-DREADD activation of $\mathrm{mPFC} \rightarrow \mathrm{DR}$ pyramidal neurons.

\section{Figure 6 Activation of GAD+ neurons probably driven by mCherry+ neurons}

(a) The percentage of GAD $+/ \mathrm{cFos}+$ neurons across all layers of the DREADD experimental group $(\mathrm{N}=7)$ and the control group $\left(\mathrm{N}^{\prime}=10\right)$. Each data point represents the average value of an animal. Data are shown as mean \pm SEM. (b) Correlation between $\% \mathrm{GAD}+/ \mathrm{cFos}+$ and $\%$ mCherry $+/ \mathrm{cFos}+$ in the $\mathrm{mPFC}$ of DREADD experimental animals. Dashed line indicates correlation close to significance. (c) Distribution of the GAD+/cFos + neurons across the layers (left) and of the GAD+/cFos-negative neurons across the layers (right). ${ }^{*} \mathrm{p} \leq 0.05$ and ${ }^{* *} \mathrm{p} \leq 0.01$ by the Mann-Whitney test.
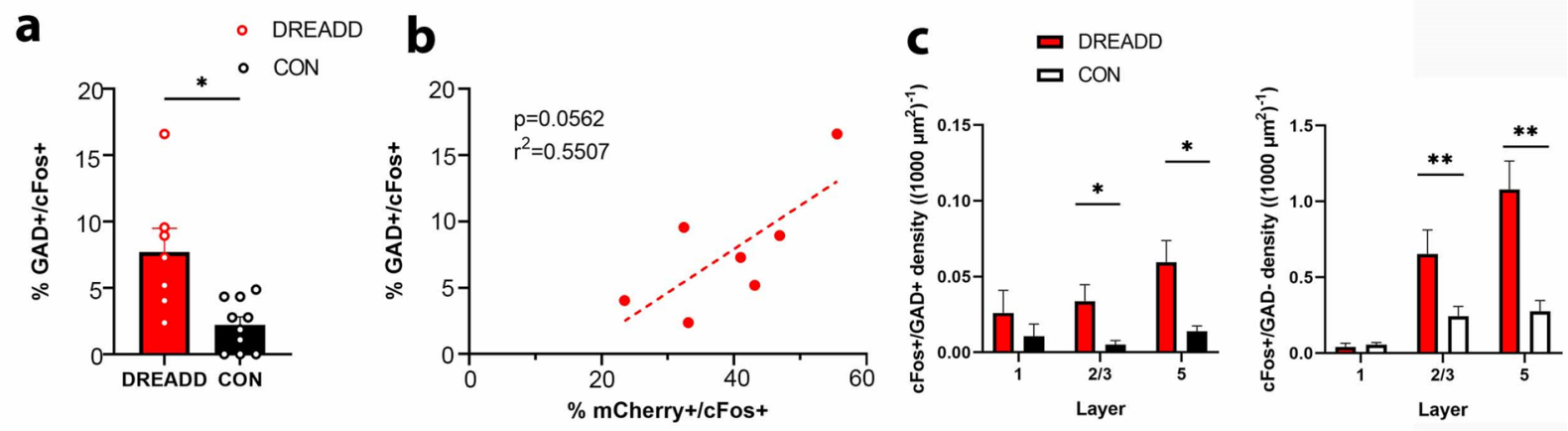


\section{Electron microscopic validation of Gq-DREADD expression in Layer 5 pyramidal neurons}

EM imaging combined with immunocytochemistry enabled ultrastructural localization of mCherry-tagged Gq-DREADDs. As shown in Fig. 7, mCherryimmunoreactivity was on the plasma membrane and in the cytoplasm of Layer $5 \mathrm{mPFC}$ pyramidal neurons' cell bodies (Fig. 7 a), apical dendrites (Fig. 7 b), and spine heads forming excitatory synapses (Fig. 7 c). Together with Fig. 3, these results confirm the expression of Gq-DREADDs and their wide localizations within Layer 5 pyramidal neurons in the mPFC.

\section{Electron microscopic analysis reveals more prevalent GABAergic innervation of mPFC $\rightarrow$ DR pyramidal cells than of neighboring Layer 5 pyramidal cells without projections to DR}

GABAergic innervation of Layer 5 pyramidal neurons in the mPFC of DREADD experimental animals was quantified in two ways: the percentage of plasma membrane covered by GABAergic terminals (\%GABA innervation) and the number of GABAergic terminals per unit plasma membrane length (GABA Terminal Density) (Fig 8 a). The quantification was done for both mCherry+ neurons and mCherry-negative pyramidal neurons in Layer 5 of mPFC. Neuronal cell bodies were verified to be pyramidal, based on the smooth contour of the nuclear membrane (White, 1989) and lack of GAD immunoreactivity. Both measurements were significantly greater for the mCherry + pyramidal neurons than for the mCherry-negative pyramidal neurons within individual DREADD experimental animals (Fig. 8 b) (paired t test, $t_{(6)}=3.723, P=0.0098$ for $\%$ GABA; paired $\mathrm{t}$ test, $t_{(6)}=6.932, P=0.0004$ for GABA Terminal Density). The greater \%GABA innervation of mCherry+ neurons was not due to differences in the lengths of individual GABAergic 
terminals (Kolmogorov-Smirnov $D=0.08214, P=0.2392$ ), but due to a significant decrease in their inter-synaptic lengths (Kolmogorov-Smirnov $D=0.1298, P=0.0102$ ) (Fig. 8 c).

Figure 7 Electron microscopic validation of the presence of Gq-DREADDs on neurons

(a) DREADD molecules labeled by silver intensified gold (SIG) particles were present on the plasma membrane (white wedges) and in the cytoplasm (need to point to it with a symbol) of Layer $5 \mathrm{mPFC}$ pyramidal neurons' cell body. The plasma membrane of an adjacent non-transfected pyramidal neuron with almost no Gq-DREADD-mCherry-immunoreactivity is shown on the right (red neuron). Scale bar $=500 \mathrm{~nm}$. (b) SIG, reflective of Gq-DREADD/mCherry that was present on the plasma membrane of an apical dendrite (qA). Scale bar $=1 \mu \mathrm{m}$. (c) SIG reflective of Gq-DREADD/mCherry that was present along the plasma membrane of an dendritic spine head (white asterisk) forming an excitatory synapse that showed the postsynaptic density indicated by the white arrowheads. Scale bar $=300 \mathrm{~nm}$. N, nucleus; Mito, mitochondria; G, Golgi apparatus; ER, endoplasmic reticulum; T, axon terminal; qA, apical dendrite.
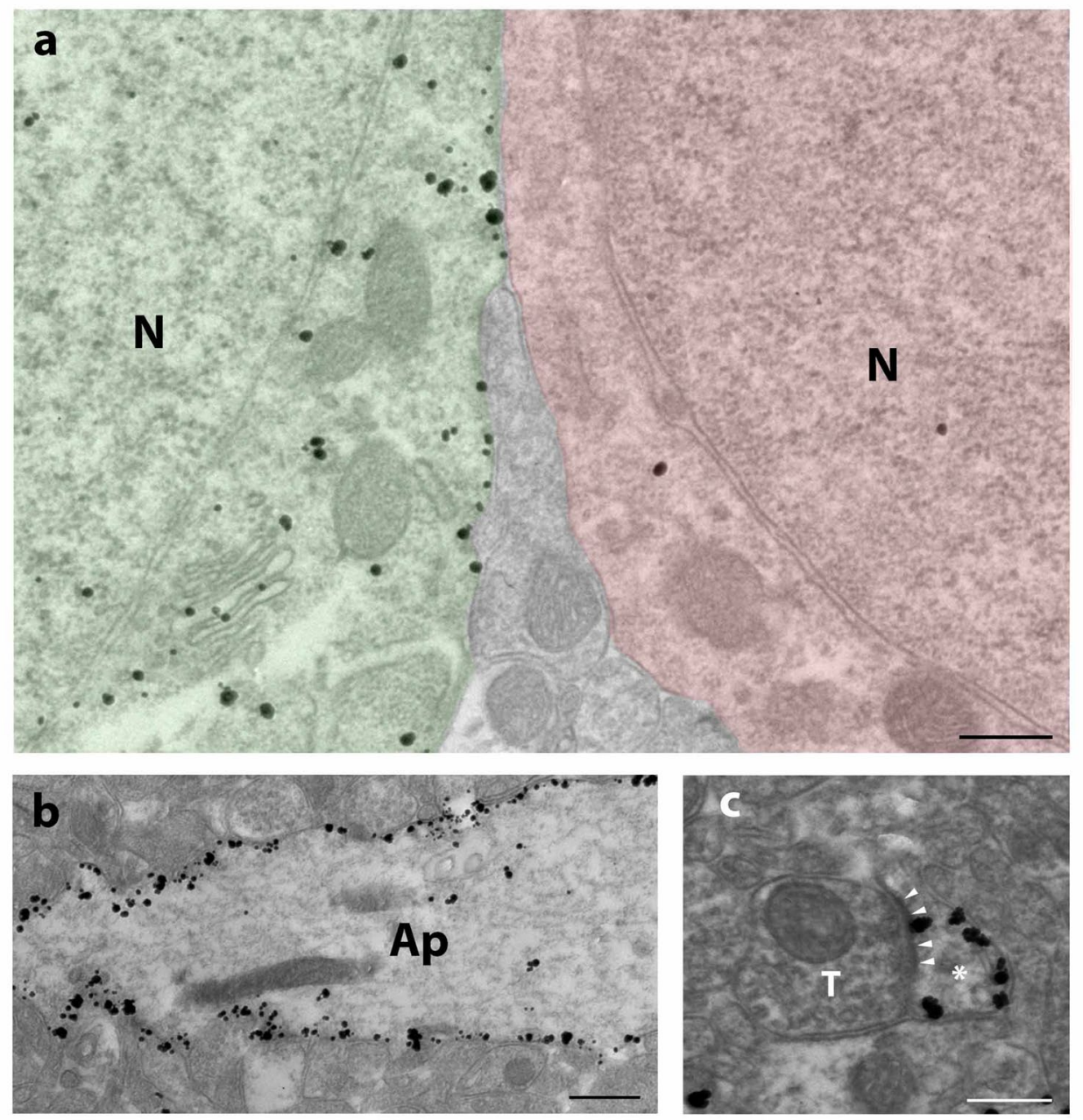
Figure 8 Electron microscopic quantification of GABAergic innervation of mCherry + and mCherry-negative neurons' cell bodies in Layer 5 of mPFC

(a)Schematic illustration for the quantification of GABAergic innervation. Left, a cartoon showing an mCherry+ pyramidal (Pyr) neuron labeled by silver intensified gold particles (black dots). The GABAergic terminals (dark triangles) from the interneurons (G-IN) are distinguished from the other terminals (light triangles) due to the HRP-DAB reaction product indicating the presence of GAD. The cartoon does not reflect the relative size of a neuron and terminals. Right, an EM image showing a portion of a silver intensified gold (white arrow) labeled neuron receiving inputs from GABAergic terminals (white asterisks) and other terminals (black asterisk). The green area covers the entire SIGlabeled neuron, the red segments indicate the GABAergic terminals aligned with the plasma membrane. The expressions for \% GABA innervation and the density of GABAergic terminals are shown on the right. Scale bar $=500 \mathrm{~nm}$. (b) Comparisons of \% GABA innervation (left panel), and the GABA terminal density (right panel), between the mCherry+ and mCherry-negative neurons. from the same DREADD experimental animal. (c) Comparisons and cumulative density funcions of the GABAergic terminal inter-synaptic lengths (right panel), and individual GABAergic terminal lengths (left panel) of the mCherry+ $(n=69)$ and mCherry-negative $(n=83)$ neurons sampled across all of the DREADD experimental animals $(\mathrm{N}=7)$. (d) Correlations between $\% \mathrm{mCherry}+\mathrm{cFos}+$ and $\% \mathrm{GABA}$ innervation (left panel), or GABAergic terminal density (right panel), of mCherry+ and mCherrynegative neurons. Each point represents the averaged quantities from an individual DREADD experimental animal. Significant correlation is shown with straight line together with its p-value and $r$ square. $* \mathrm{p} \leq 0.05, * * \mathrm{p} \leq 0.01, * * * \mathrm{p} \leq 0.001$

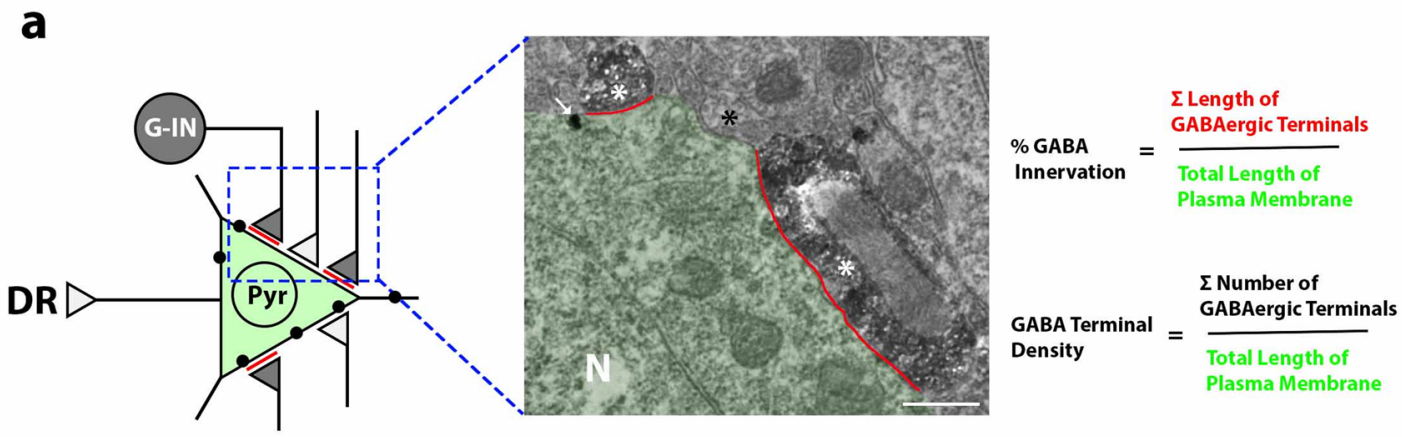

b

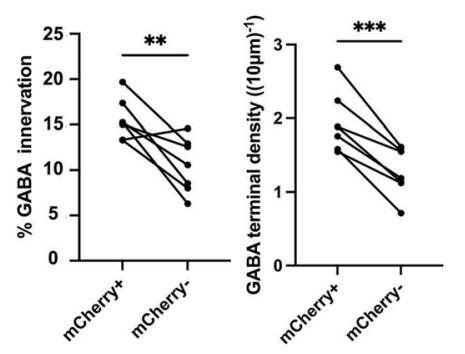

d

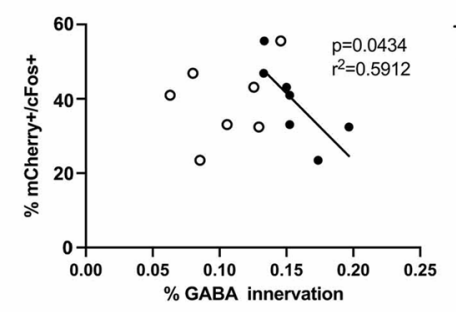

C
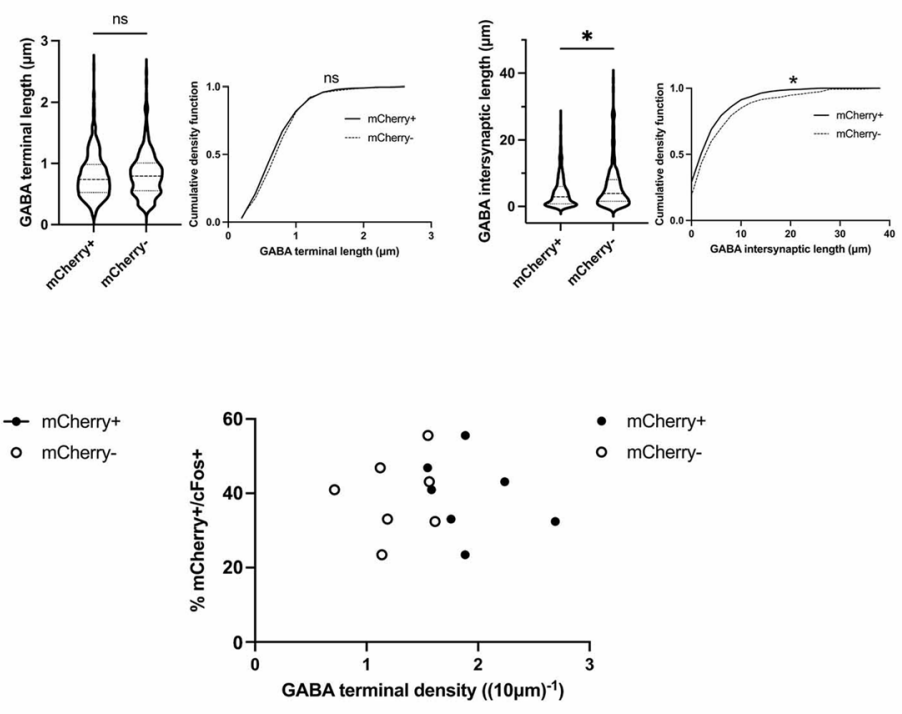


\section{Electron microscopic analysis reveals more prevalent GABAergic innervation of mPFC $\rightarrow$ DR pyramidal cells than of neighboring Layer 5 pyramidal cells without projections to $\mathrm{DR}$}

GABAergic innervation of Layer 5 pyramidal neurons in the mPFC of DREADD experimental animals was quantified in two ways: the percentage of plasma membrane covered by GABAergic terminals (\%GABA innervation) and the number of GABAergic terminal per unit plasma membrane length (GABA Terminal Density) (Fig 8 a). The quantification was done for both mCherry + neurons and mCherry-negative pyramidal neurons in Layer 5 of mPFC. Neuronal cell bodies were verified to be pyramidal, based on the smooth contour of the nuclear membrane (White, 1989) and lack of GAD immunoreactivity. Both measurements were significantly greater for the mCherry + pyramidal neurons than for the mCherry-negative pyramidal neurons within individual DREADD experimental animals (Fig. 8 b) (paired t test, $t_{(6)}=3.723, P=0.0098$ for $\%$ GABA; paired $\mathrm{t}$ test, $t_{(6)}=6.932, P=0.0004$ for GABA Terminal Density). The greater \%GABA innervation of mCherry+ neurons was not due to differences in the lengths of individual GABAergic terminals (Kolmogorov-Smirnov $D=0.08214, P=0.2392$ ), but due to a significant decrease in their inter-synaptic lengths (Kolmogorov-Smirnov $D=0.1298, P=0.0102$ ) (Fig. 8 c).

\section{Correlations between immunofluorescently labeled cells and GABAergic innervation}

For the DREADD experimental animals, \%mCherry + cFos + (immunofluorescent data) was significantly and negatively correlated with \%GABA innervation of mCherry+ pyramidal cells (EM data) $\left(r^{2}=0.5912, P=0.0434\right)$ but not with the values of $\%$ GABA innervation of mCherry-negative neurons (Fig. 8 d). This indicates that those individuals with relatively stronger GABAergic innervation of $\mathrm{mPFC} \rightarrow \mathrm{DR}$ pyramidal neurons exhibited greater suppression of the $\mathrm{mPFC} \rightarrow \mathrm{DR}$ pathway. In contrast, the correlation of the GABA 
terminal density with \%mCherry $+/ \mathrm{cFos}^{+}$was not significant (Fig. $8 \mathrm{~d}$ ). This indicates that the percent coverage of the pyramidal neurons' cell bodies played a more significant role than the number of GABAergic terminals in modulating neuronal activity and that this GABAergic modulation was specific to the subpopulation of Layer 5 pyramidal neurons with projections to DR and driven by $\mathrm{C} 21$.

\section{Correlation analysis of immunocytochemical data to feeding behaviors during FR}

To address whether the circuits involving the $\mathrm{mPFC} \rightarrow \mathrm{DR}$ were related to the feeding behaviors of mice undergoing ABA, correlation analyses of data obtained by EM (\%GABA innervation of mCherry + and mCherry-negative pyramidal cells), immunofluorescence $\left(\% \mathrm{mCherry}+/ \mathrm{cFos}+, \% \mathrm{GAD}+/ \mathrm{cFos}^{+}\right)$and food intake of the DREADD experimental animals were performed. As shown in Fig. 9, food intake on FR days of ABA1 did not correlate with EM or immunofluorescence data.

During ABA2, \%mCherry $+/ \mathrm{cFos}+$ correlated significantly and positively with food intake on FR2, the day when $\mathrm{C} 21$ was injected to activate the $\mathrm{mPFC} \rightarrow \mathrm{DR}$ pathway $\left(r^{2}=0.6091, P=0.0384\right)$ (Fig. 10 a). These variables showed no correlation on FR1, FR3 or FR4 (Fig. 10 a). The positive correlation conforms to the previous literature regarding DR's roles in feeding (Bendotti et al., 1986; Fletcher \& Davies, 1990; Nectow et al., 2017) as well as the connectivity between mPFC and DR (Jankowski \& Sesack, 2004).

While $\% \mathrm{GAD}+/ \mathrm{cFos}+$ appeared to be unrelated to feeding on FR2 (Fig. 10 b), \%GABA innervation only of mCherry+ (Fig. 10 c) (i.e., not the mCherry-negative pyramidal neurons in Layer 5, Fig. 10 d) exhibited a very significant negative correlation with the food intake specifically on FR2, when C21 was administered $\left(r^{2}=0.8429, P=0.0035\right)$. 
Fig 9 Correlations of feeding behaviors during ABA1 with the IF and EM data Correlations between the food intake during the first ABA and \%mCherry $+\mathrm{cFos}+(\mathrm{a}), \% \mathrm{GAD}+\mathrm{cFos}+(\mathrm{b})$, $\% \mathrm{GABA}$ of mCherry+ neurons (c) and \%GABA of mCherry-negative neurons (d).

a
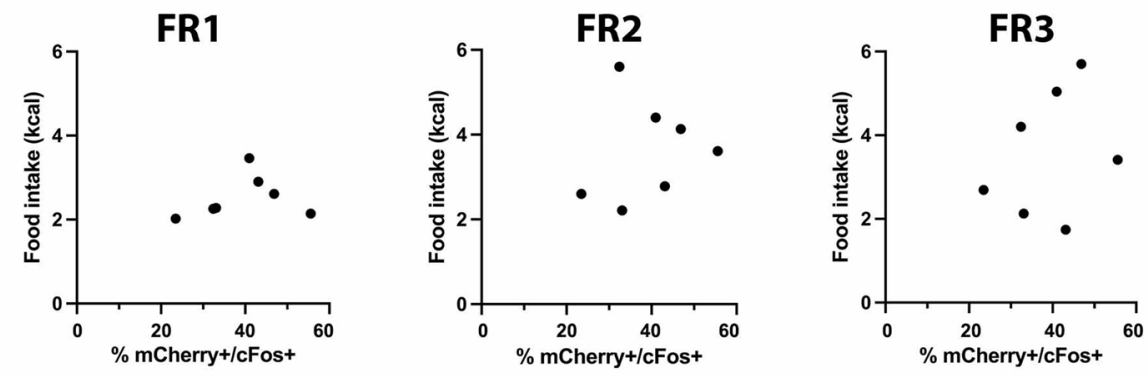

b
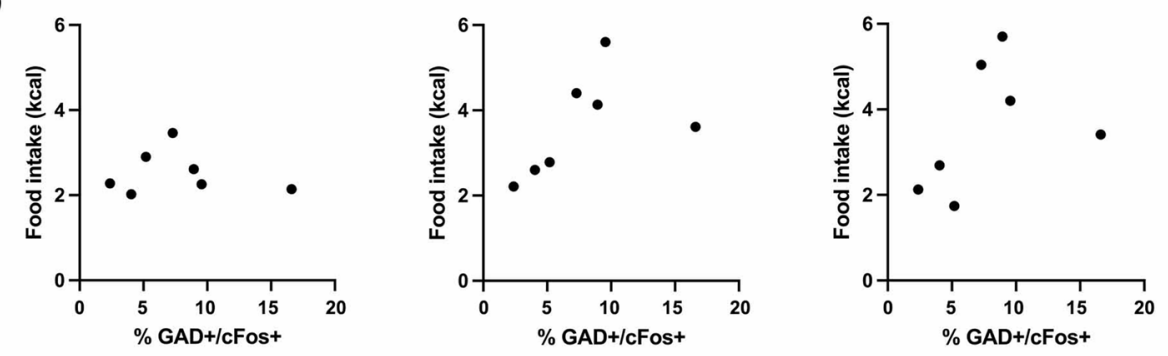

C
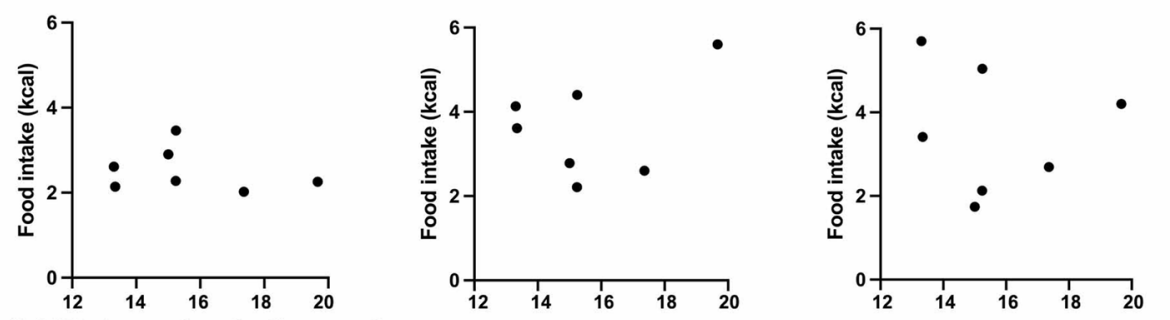

d
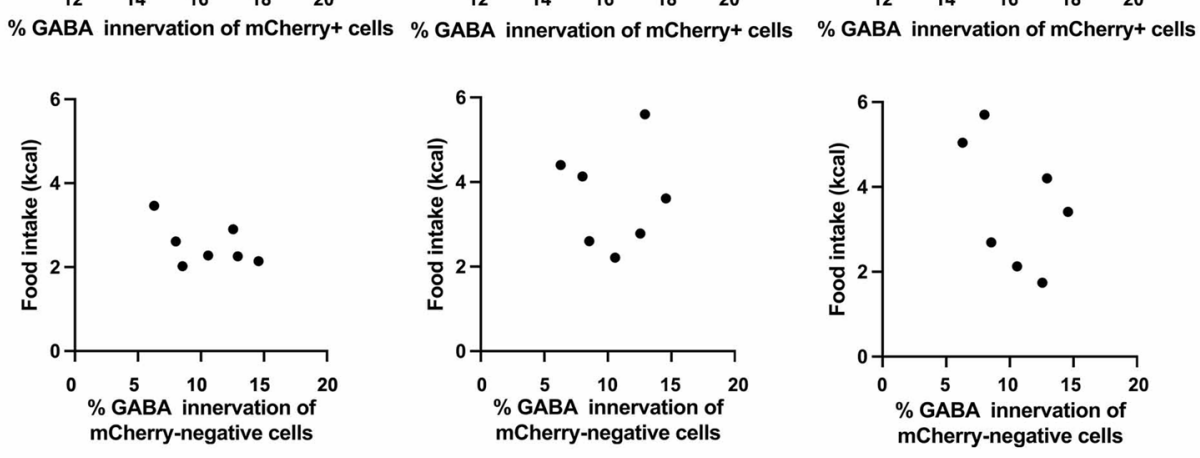
Fig 10 Feeding behavior during ABA2 and their correlations with the IF and EM data (C) Correlations between the food intake during the second $\mathrm{ABA}$ and $\% \mathrm{mCherry}+/ \mathrm{cFos}+(\mathrm{a})$, $\% \mathrm{GAD}+/ \mathrm{cFos}+(\mathrm{b}), \% \mathrm{GABA}$ innervation of mCherry+ neurons (c) and \%GABA innervation of mCherry-negative neurons (d). Significant correlations were indicated by straight lines together with their p-values and r-squares. Correlation close to significance was indicated by dashed line.

a
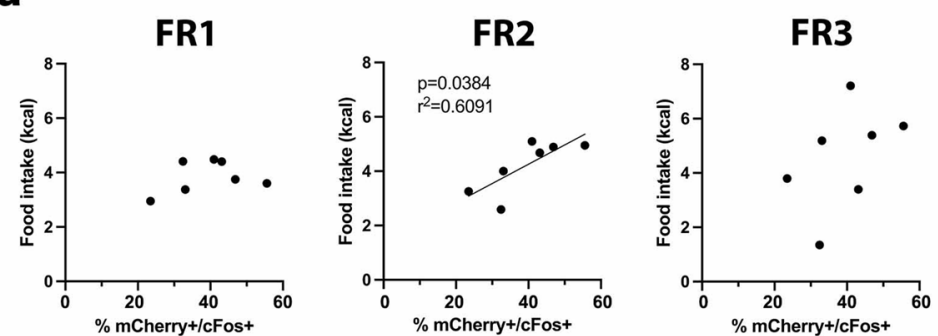

FR4

b
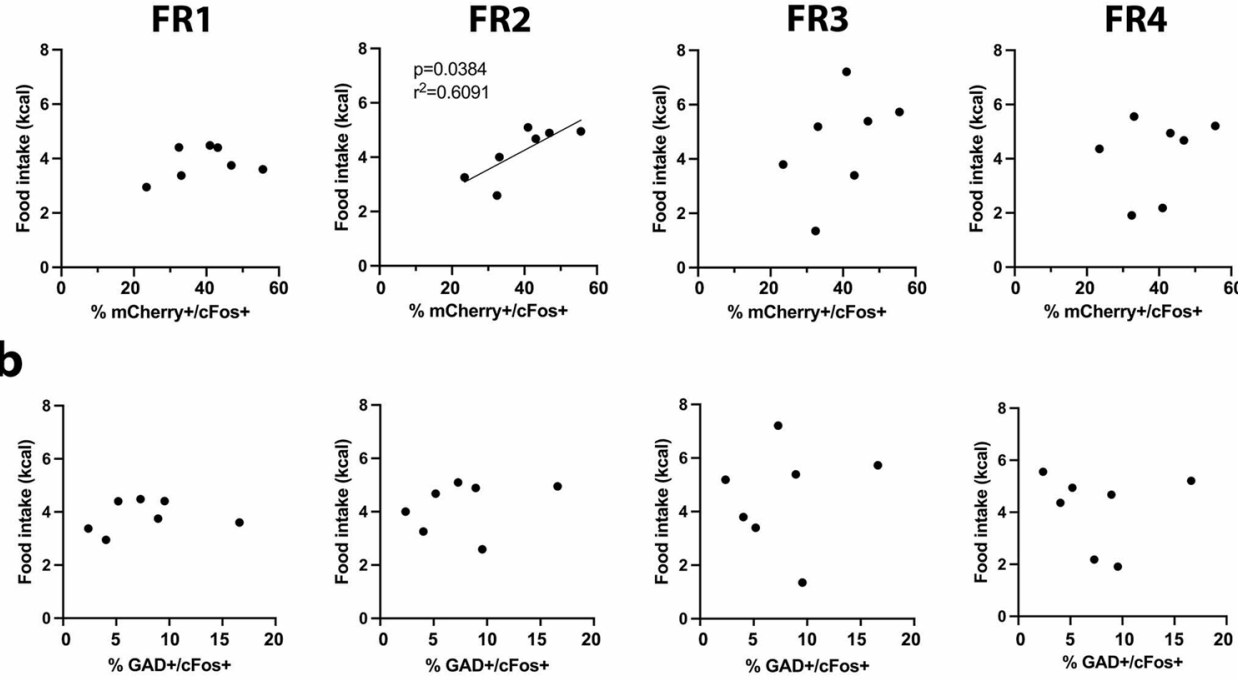

C
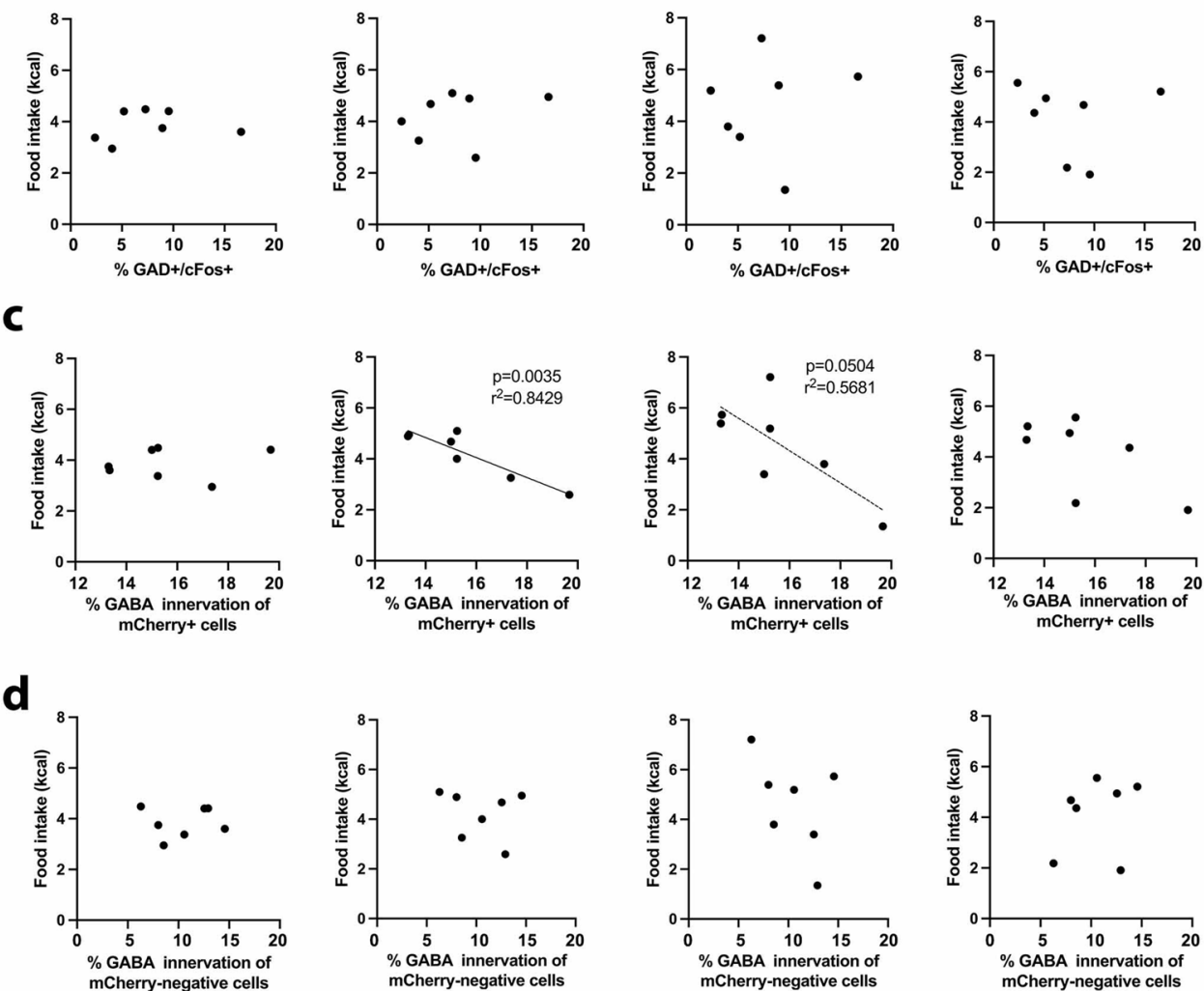


\section{Discussion}

This project combined chemogenetic stimulation with confocal and EM imaging to elucidate the connectivity of $\mathrm{mPFC} \rightarrow \mathrm{DR}$ pyramidal neurons and their roles in regulating feeding behaviors of mice in the ABA model. Contrary to our initial expectation, direct activation or inhibition of the mPFC $\rightarrow$ DR pathway by DREADDs did not yield an effect that could be detected as a group mean difference, relative to control animals' behavior without chemogenetic modulation. Closer inspection revealed that this was due to the wide variance in behavior that obscured the neuromodulatory effects of DREADDs upon individuals. By pursuing correlative analysis of confocal data of individual animals, we revealed that individuals with greater DREADD-mediated activation of $\mathrm{mPFC} \rightarrow \mathrm{DR}$ pyramidal cells also exhibited greater food consumption (Fig 10) and activation of GABA-INs (Fig 6). Moreover, analysis of the EM data on GABAergic axo-somatic innervation, relative to food intake revealed a strong negative correlation: the more prevalent the GABAergic innervation of $\mathrm{mPFC} \rightarrow \mathrm{DR}$ pyramidal neurons, the more suppressed were an individual's food intake. These findings, together, reveal that $\mathrm{mPFC}$ does modulate feeding behavior within the context of ABA, with stronger GABAergic inhibition of the corticofugal pathway projecting to the DR resulting in reduced feeding.

\section{The mPFC microcircuit activated by chemogenetic modulation of the mPFC $\rightarrow$ DR pyramidal neurons}

Previous studies successfully modulated the mPFC $\rightarrow$ DR pathway through two approaches: Injecting currents into the DR-projecting pyramidal neurons in the $\mathrm{mPFC}$ (Celada, Puig, Casanovas, Guillazo, \& Artigas, 2001), or optogenetically stimulating axons within DR with cell bodies of origin in the mPFC (Warden et al., 2012). The chemogenetic modulation of the $\mathrm{mPFC} \rightarrow$ DR pathway was like the approach of Celada et al. (Celada et al., 
2001) in having a greater chance of recruiting other neurons within mPFC as well as other brain regions. Indeed, in our study, we showed that the direct chemogenetic activation of the DR-projecting pyramidal neurons in Layer 5 of mPFC also significantly boosted the activities of Layer 2/3 neurons, which were GABAergic and pyramidal (Fig. 5 c). This means that microcircuits from DR-projecting Layer 5 pyramidal neurons feed back to Layers $2 / 3$ of $\mathrm{mPFC}$, in addition to the canonical cortical circuit with projections from Layers $2 / 3$ pyramidal neurons to Layer 5 pyramidal neurons (Dhruv, 2015). While this type of feedback pathway from deeper to superficial layers as well as their functional implications are wellstudied in the visual cortex (reviewed in (Capone et al., 2016)), such circuits are less studied in the mPFC. At least some of the activated neurons in Layers $2 / 3$ were pyramidal neurons which, in turn, are known to project to dorsal and ventral striatum (Jankowski \& Sesack, 2004) and enhance wheel running during FR within the context of ABA (Santiago et al., 2021). Those activities may exert complicated effects that were intermingled with the roles of the DR-projecting neurons in regulating feeding of our ABA animals.

If the correlation between $\% \mathrm{GAD}+/ \mathrm{cFos}+$ and $\% \mathrm{mCherry}+/ \mathrm{cFos}+$ had been negative, this would have implied that firing of GABA-INs controlled excitability of mPFC $\rightarrow$ DR pyramidal cells. Instead, the correlation between these two variables was positive (Fig. $6 \mathrm{c}$ ). This supports an alternative view - namely that firing of GABA-INs was driven by the $\mathrm{mPFC} \rightarrow \mathrm{DR}$ neurons. The idea that DR-projecting pyramidal neurons drive the GABA-INs across layers in $\mathrm{mPFC}$ conforms to the previous findings of local GABAergic microcircuits in mPFC (Sun et al., 2019). Activation of GABA-INs upon DREADD stimulation of the $\mathrm{mPFC} \rightarrow \mathrm{DR}$ pathway might be a way for the animals to avoid hyperactivity and excitotoxicity.

The current study showed that the overall activity of DR-projecting neurons could be affected significantly and negatively by the extent of GABAergic innervation that they 
received (Fig. 8 d). This illustrates a strong link between pyramidal neurons' firing and their ultrastructure - specifically, the axo-somatic synapses supporting negative feedback. Since greater firing of GABA-INs did not result in suppression of $\mathrm{mPFC} \rightarrow \mathrm{DR}$ pyramidal neurons, these findings, together, reveal that excitability of Layer 5 pyramidal neurons is dictated more by ultrastructure of axo-synaptic inputs formed by GABAergic axon terminals than by the firing properties of the GABA-INs.

\section{A novel synaptic feature of $\mathrm{mPFC} \rightarrow \mathrm{DR}$ pyramidal neurons is revealed by EM-ICC: Prevalent GABAergic innervation}

The EM-immunocytochemical approach enabled us to distinguish the DR-projecting versus non-DR-projecting pyramidal neurons in Layer 5 of mPFC based on the immunoreactivity of pyramidal cell bodies to mCherry. Previous studies had shown that ABA could cause an enlargement in the GABAergic coverage of the Layer 5 pyramidal neurons in mPFC (Chen, Wable, Chowdhury, \& Aoki, 2016). The current study extends that finding by showing that the DR-projecting neurons receive more GABAergic innervation than other pyramidal neurons in Layer 5 of mPFC that do not project to DR (Fig. 8 b). This could mean that the enlargement of GABAergic terminals in the mPFC of ABA animals revolves the DRprojecting subgroup, especially. It is also likely that the DR-projecting neurons are more resistant to the chemogenetic stimulation due to the stronger inhibitory feedback that they receive.

Whether the greater GABAergic innervation is an intrinsic property of the DRprojecting neurons or reflects plastic change caused by the Gq-DREADD-induced hyperactivity remains to be investigated. We think the former is more likely. This thought is based on an earlier observation that Layer 5 pyramidal neurons of $\mathrm{mPFC}$ without projections to dorsal striatum (DS) exhibited greater GABAergic innervation than the corticostriatal 
neurons (CSt) labeled retrogradely from DS, even though these $\mathrm{mPFC} \rightarrow \mathrm{DR}$ pyramidal cells were not designed to be modulated by DREADD ligands (Santiago et al., 2021). This suggests that there are innate differences in GABAergic innervation across subpopulations of Layer 5 pyramidal neurons depending on their projection patterns. Although both the PT (pyramidal tract) and IT (intratelencephalic) subpopulations of Layer 5 neurons in motor and somatosensory cortex project to DS and are thus considered CSt (Reiner, Jiao, Del Mar, Laverghetta, \& Lei, 2003; Shepherd, 2013), the extent of mPFC's PT collaterals to DS (which would include the mPFC $\rightarrow$ DR pyramidal cells studied here) is much less than for the mPFC's IT subpopulation of CSt pyramidal cells (Anastasiades \& Carter, 2021). Both our previous (Santiago et al., 2021) and current ultrastructural analyses indicate that the $\mathrm{mPFC} \rightarrow \mathrm{DR} / \mathrm{PT}$ cells of Layer 5 belong to the subpopulation of neurons in Layer $5 \mathrm{of} \mathrm{mPFC}$ with greater GABAergic innervation. This agrees with electrophysiological studies indicating that the PT subpopulation of CSt in Layer 5 of mPFC are more strongly inhibited than are the IT-CSt neurons in Layer 5 (Anastasiades \& Carter, 2021; Lee et al., 2014).

\section{Chemogenetic modulation of the mPFC $\rightarrow$ DR pathway enhances food intake}

Although activation or inhibition of the mPFC-DR pathway by DREADDs did not significantly alter the group mean average of food intake of mice in the ABA model compared to control animals lacking DREADD gene expression (Fig. 2 d), correlation analysis performed for the experimental animals showed that individuals with greater activities among the DR-projecting pyramidal neurons exhibited greater food intake. Notably, the correlation between food intake and neuronal firing of $\mathrm{mPFC} \rightarrow \mathrm{DR}$ pyramidal neurons appeared only on FR2 of ABA2, and not FR1, 3 or 4 (Fig. 10 a). Considering that C21 was injected only on FR2 to activate the $\mathrm{mPFC} \rightarrow \mathrm{DR}$ pathway, the following explanation is proposed: On FR2, activity of the $\mathrm{mPFC} \rightarrow \mathrm{DR}$ pathway may already have been very high due 
to animals' active coping with starvation stress (Amat et al., 2005). Under this circumstance, activation of the excitatory DREADDs by C21 may not have been able to boost the activity of the $\mathrm{mPFC} \rightarrow \mathrm{DR}$ pyramidal neurons any further. Instead, C21 could have generated differential activities of the DR-projecting pyramidal neurons (\%mCherry $+/ \mathrm{cFos}+)$ through differential GABAergic inhibition. However, $\% \mathrm{GAD}+/ \mathrm{cFos}+$ did not correlate with food intake on any day of ABA2, even on FR2 when C21 was injected. (Fig. 10 b). This is probably because individual differences in the inhibitory effects caused by the activation of the GABA-INs in mPFC following C21 injection was not specific to the DR-projecting neurons, but acted on a wide range of neurons.

\section{Individual differences in food intake is influenced by ultrastructural differences in} GABAergic axo-somatic synapses on $\mathrm{mPFC} \rightarrow$ DR pyramidal neurons more than by

\section{GABA neuronal firing}

Importantly, the differential GABAergic inhibition appears to have resulted from differential levels in GABAergic neurons' axonal structure - i.e., the extent of axo-somatic innervation of $\mathrm{mPFC} \rightarrow \mathrm{DR}$ pyramidal neurons, rather than differential firing of GABA-INs. This idea is derived from the observation that correlation was evident between food intake and the extent of GABAergic innervation (\%GABA), specifically of pyramidal neurons with projections to DR (Fig. $10 \mathrm{c}$ ) and not the pyramidal neurons lacking projections to DR (Fig. $10 \mathrm{~d}$ ), further supporting the idea that the $\mathrm{mPFC} \rightarrow \mathrm{DR}$ pathway is involved especially in the feeding behaviors of mice. The contrasting lack of correlation between the $\% \mathrm{GAD}+/ \mathrm{cFos}+$ and food intake is indication that the strength of GABAergic inhibition is influenced more by axonal structure than by the firing pattern of GABAergic neurons.

The correlation between $\%$ GABA innervation of mCherry+ pyramidal neurons and food intake is negative (Fig. $10 \mathrm{c}$ ), while the correlation between $\mathrm{mCherry}+/ \mathrm{cFos}+$ and food 
intake is positive (Fig. 10 a). These two correlations with opposite valences agree with the notion that excitatory outflow from the mPFC to DR stimulates feeding and that synaptic plasticity underlying the gain of resilience to ABA (measured based on increased food intake) may be the retraction of GABAergic innervation of $\mathrm{mPFC} \rightarrow \mathrm{DR}$ pyramidal neurons.

Since activation of serotonergic neurons in the DR suppresses feeding (Nectow et al., 2017), the dominant targets of $\mathrm{mPFC} \rightarrow \mathrm{DR}$ in DR may be the GABA-IN that inhibit firing of the serotonergic neurons. Such direct pathway from pyramidal neurons in mPFC to GABAIN in DR has been elucidated by EM (Jankowski \& Sesack, 2004), this may be an alternative or additional site of synaptic plasticity of animals that gain resilience following ABA induction. Further analysis of the activity of GABA-INs in the DR following chemogenetic activation of the $\mathrm{mPFC} \rightarrow \mathrm{DR}$ pyramidal neurons promises to yield data testing this hypothesis.

\section{Lack of effect of SalB/KORD}

If the ceiling effect was accountable for our observation of food intake on FR2, then SalB injection on FR4 should have significantly decreased the activity of the $\mathrm{mPFC} \rightarrow \mathrm{DR}$ pathway and thus decreased the food intake of mice. However, this was not observed. A possible explanation for this is that further decrease in food intake on FR4 when the animals had already been food restricted for three days and had lost a significant amount of body weight would put them under severe danger of starving to death. Under this circumstance, other players such as the $\mathrm{mPFC} \rightarrow$ lateral hypothalamus pathway which is also capable of regulating feeding (Petrovich, Holland, \& Gallagher, 2005) may have interfered with SalB modulation, thereby saving the animals from death.

\section{Conclusion}


We dissected the circuits in $\mathrm{mPFC}$ that involved the DR-projecting pyramidal neurons in Layer 5 through immunofluorescence and EM immunocytochemistry. Summarized in Fig. 11 , we show that $\mathrm{mPFC} \rightarrow \mathrm{DR}$ pyramidal neurons send outputs not only to DR but also to Layers $2 / 3$ pyramidal neurons and GABA-INs across layers. Thus, DREADD ligands modulate activity of a multi-synaptic mPFC circuitry rather than a single population of neurons. Through correlation analysis, we have gathered data supporting the hypothesis that the $\mathrm{mPFC} \rightarrow \mathrm{DR}$ pathway is a high-order regulator of feeding for ABA mice and possibly also for AN patients, favoring them to overcome the maladaptive behavior of FR, when activated. Conversely, stronger the inhibition of this pathway, the more food restrictive individuals may become. This finding differs sharply from another recent finding from our lab, demonstrating that activation of pyramidal neurons of the $\mathrm{mPFC}$ projecting to dorsal striatum $(\mathrm{mPFC} \rightarrow \mathrm{DS})$ exacerbates the food restriction-evoked wheel running of ABA mice (Santiago et al., 2021). On the question of whether the mPFC is the site for generating the decision to eat (i.e., adaptive for survival) or to run (i.e., maladaptive for mice in captivity), one factor influencing this decision may be the ratio of GABAergic innervations across the two parallel pathways. Within Layer 5 of ABA mice, the $\mathrm{mPFC} \rightarrow$ DR pyramidal neurons receive more GABAergic innervation than do the neurons projecting elsewhere, presumably including the mPFC $\rightarrow$ DS pyramidal neurons. This GABAergic innervation pattern may underlie individual differences in vulnerability to ABA, defined both as heightened wheel running (due to weaker inhibition of the $\mathrm{mPFC} \rightarrow \mathrm{DS}$ pathway) and stronger suppression of feeding (due to the stronger inhibition of the $\mathrm{mPFC} \rightarrow \mathrm{DR}$ pathway). For animals that have experienced $\mathrm{ABA}$, our findings predict that suppression of GABAergic activity in the $\mathrm{mPFC}$ would reduce $\mathrm{ABA}$ vulnerability. This idea is undergoing testing through chemogenetic modulation of GABAINs in the mPFC. 
Figure 11 Summary of the proposed microcircuits in mPFC that involve the DR-projecting pyramidal neurons in this study

The DR-projecting neurons (green) are distinguished from the others because of the mCherry-tagged DREADDs on their membranes (black dots). While those neurons project to DR, they may also send outputs to the Layer $2 / 3$ pyramidal neurons and the GABAergic neurons across layers in $\mathrm{mPFC}$. Those DR-projecting neurons receive greater GABAergic innervation than other Layer 5 pyramidal neurons, which could make them more resistant to the chemogenetic excitation. Pyr, pyramidal neuron; G-IN, GABAergic interneuron; DRN ${ }^{\mathrm{GABA}}$, GABAergic neurons in DR; DRN ${ }^{5-\mathrm{HT}}$, serotonergic neuron in DR.

DR mPFC

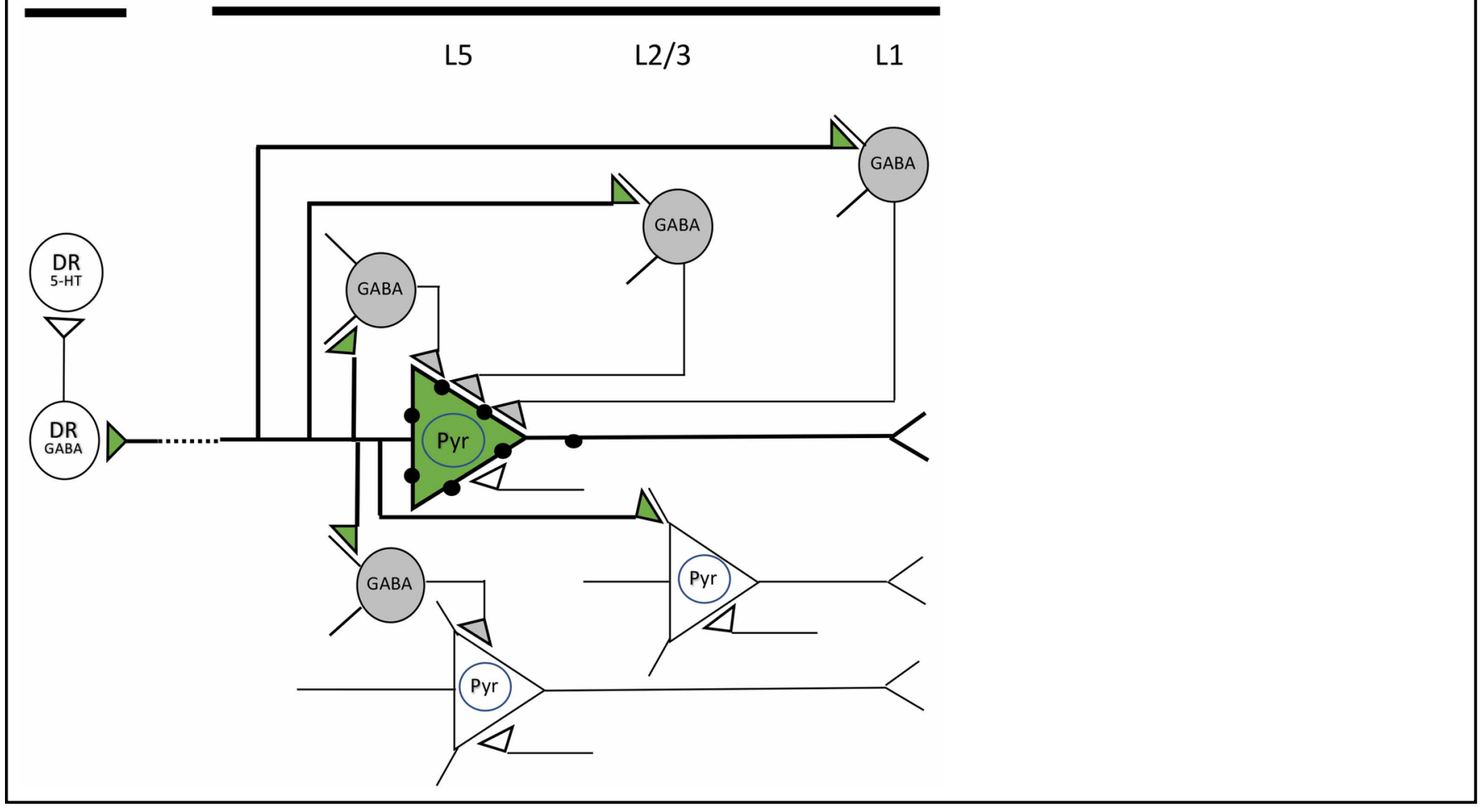


Research on AN has uncovered the complexity of this mental illness at both the biological (Bulik, Slof-Op't Landt, van Furth, \& Sullivan, 2007) and the socio-cultural (Garner \& Garfinkel, 1980; Polivy \& Herman, 2002) levels. Though AN is not a purely monogenic mental illness such as Huntington's Disease, modern studies provide substantial evidence pointing to the genetic risk factors involved in it. For example, AN tends to aggregate in families (Holland, Sicotte, \& Treasure, 1988; Strober, Freeman, Lampert, Diamond, \& Kaye, 2000), and an AN-susceptibility gene is proposed to be located on chromosome 1 (Grice et al., 2002). Besides, AN tends to affect females more than males (Smink, van Hoeken, Oldehinkel, \& Hoek, 2014; Timko, DeFilipp, \& Dakanalis, 2019), suggesting the involvement of sex steroids or chromosomes in the development of AN (Wable, Chen, Rashid, \& Aoki, 2015). The sex difference could also be due to greater sociocultural stressors placed on female adolescents, versus male (Collison \& Barnier, 2019; Garner \& Garfinkel, 1980; Polivy \& Herman, 2002; Thompson \& Stice, 2001). Furthermore, recent research could even trace the etiology of AN to an epigenetic level (Hubel, Marzi, Breen, \& Bulik, 2019). In addition to the biological risk factors, profound familial influences and socio-cultural factors such as the culture of abundance and peer influences also contribute to the cause of AN. The central role played by structural differences in GABAergic axonal arbors in generating individual differences in resilience/vulnerability to ABA highlight the importance of probing for genes linked to the GABAergic system and for designing pharmacological treatments that target GABA receptor activity for the treatment of AN. 


\section{Figure Legends}

\section{Figure 1 ABA1 timeline and behavior}

(a) Schematic illustration of the first ABA induction and the daily food restriction (FR)

schedule. See Method section for the details of the ABA1 induction. FAA: food anticipatory activity; FA: food availability; PP: post prandial period. (b) individual daily wheel count (WCT); (c) WCT during ABA; (d) Body weight; (e) Food intake during the pre-ABA and the three days of FR. The average daily food intake during the two days before the onset of FR on P41 was used as the Pre-ABA baseline food intake. The average daily food intake during the two days after ABA, on P44, was used as the post-ABA food intake.

\section{Figure 2 ABA2 timeline and behavior}

(a) Schematic illustration of the second ABA induction. See Method section for the details of the second ABA induction. (b) WCT; (c) body weight; (d) Food intake during the pre-ABA and the four days of food restriction. * indicates $p \leq 0.05$.

Figure 3 Confirmation of DREADD expression in the $\mathrm{mPFC} \rightarrow \mathrm{DR}$ neurons and representative triple IF confocal images of $\mathrm{mPFC}$ neurons

(a) Schematic of the stereotaxic injection of AAVrg-EBFP-Cre into DR and of the credependent DREADD-AAV into mPFC.

(b) Verification of AAVrg-EBFP-Cre deposition into DR. Note that the region with the highest density of EBFP-immunoreactivity is in the DR. ca=cerebral aqueduct;PAG=periaqueductal grey; $\mathrm{mlf}=$ medial longitudinal fasciculus. $\mathrm{Bar}=500 \mu \mathrm{m}$.

(c) Verification of Gq-DREADD-mCherry expression in Layer 5 pyramidal neurons of the mPFC. mCherry-immunoreactivity is prevalent within cell bodies in Layer 5, apical 
dendrites traversing across Layers $2 / 3$ and branching in Layer 1 . The section was dually labeled using anti-cFos, resulting in immunoreactivity of nuclei of the same population of pyramidal neurons. Bar $=100 \mu \mathrm{m}$.

(d) Verification of Gq-DREADD-mCherry expression in puncta of DR. Vibratome sections containing DR were processed in parallel with sections containing mPFC that is shown in panel c. Puncta reflecting mCherry-immunoreactive axon terminals are evident in DR, ventral (filled arrowheads) and lateral to the cerebral aqueduct. Nuclei immunoreactive for cFos are also evident (open arrows) in DR.

(e) Verification of Gq-DREADD-mCherry expression in axons coursing within fiber bundles of the internal capsule (open arrowheads) and varicose axonal processes (filled arrowheads) in dorsal striatum. Calibration bar $=50 \mu \mathrm{m}$ and applies to panels $\mathrm{d}$ and $\mathrm{e}$.

(f) Overview of the section showing mCherry (green), cFos (red) and GAD (blue) immunofluorescence. Note that most of the mCherry signals were contained in the prelimbic (PL) and infralimbic (ILA) areas, which constitute major parts of mPFC. ACA, anterior cingulate area; fa, anterior forceps. Scale bar $=170 \mu \mathrm{m}$. (g) Laminar distribution of mCherry, cFos and GAD immunolabeling. Layers were identified according to Allen Brain Atlas based on the distance to pia: Layer 1, 0-125 $\mu \mathrm{m}$; Layers $2 / 3,125-325 \mu \mathrm{m}$; Layer 5, 325-525 $\mu \mathrm{m}$; Layer $6,>525 \mu \mathrm{m}$. Scale bar $=50 \mu \mathrm{m}$; (h) Representative image of an mCherry $+/ \mathrm{cFos}^{+}$ neuron (filled arrowhead), mCherry+/cFos-negative neurons (open arrowheads), and an mCherry-negative/cFos + neuron (arrow). Scale bar $=25 \mu \mathrm{m}$; (i) Representative image of a $\mathrm{GAD}+/ \mathrm{cFos}+$ neuron (filled arrowhead), a GAD $+/ \mathrm{cFos}-$ negative neuron (open arrowhead), and an mCherry+ dendrite with spines (asterisk). Scale bar $=10 \mu \mathrm{m}$.

Fig 4 Laminar distributions of mCherry+, GAD+ and cFos+ neurons 
(a) The same window within ILA from a DREADD experimental animal captured by GAD, mCherry and cFos immunofluorescence channels, respectively. The pial surface is located at the very bottom of the image. Scale bars $=50 \mu \mathrm{m}$. (b) The fraction of mCherry + and cFos + neurons distributed along laminae for the DREADD experimental group $(\mathrm{N}=7)$ and the control group $\left(\mathrm{N}^{\prime}=10\right)$. (c) The fraction of GAD+ neurons distributed along the laminae for the DREADD experimental group $(\mathrm{N}=7)$ and the control group $\left(\mathrm{N}^{\prime}=4\right)$. Each data point represents the relative fraction of the indicated cell type within a $50 \mu \mathrm{m}$ bin centered at the position specified by its $\mathrm{x}$-coordinate. Data are shown as mean $\pm \mathrm{SEM}$.

\section{Figure 5 cFos + neuron density across the laminae}

(a) Representative confocal images showing the cFos + neurons in PL along the laminae of a DREADD experimental animal and a control animal. The pia surface is located at the very bottom of the image. Scale bars $=50 \mu \mathrm{m}$. (b) The density of cFos + neurons across the laminae. Each data point was represented by the density of cFos + neurons in a $50 \mu \mathrm{m}$ high window centered at the position specified by its $\mathrm{x}$-coordinate. The width of the windows varied from image to image, but was typically around 300-400 $\mu \mathrm{m}$, covering PL and/or ILA.

Data are shown as mean \pm SEM. (c) The density of cFos + neurons by layers. Layer categorization was as described in Figure 3. $* \mathrm{p} \leq 0.05, * * \mathrm{p} \leq 0.01, * * * \mathrm{p} \leq 0.001, * * * * \mathrm{p} \leq$ 0.0001 by the Mann-Whitney test.

\section{Figure 6 Activation of GAD+ neurons probably driven by mCherry+ neurons}

(a) The percentage of GAD $+/ \mathrm{cFos}+$ neurons across all layers of the DREADD experimental group $(\mathrm{N}=7)$ and the control group $\left(\mathrm{N}^{\prime}=10\right)$. Each data point represents the average value of an animal. Data are shown as mean \pm SEM. (b) Correlation between $\% \mathrm{GAD}+/ \mathrm{cFos}+$ and $\%$ mCherry $+/ \mathrm{cFos}^{+}$in the mPFC of DREADD experimental animals. Dashed line indicates correlation close to significance. (c) Distribution of the GAD $+/ \mathrm{cFos}+$ neurons across the 
layers (left) and of the GAD $+/ \mathrm{cFos}-$ negative neurons across the layers (right). ${ }^{*} \mathrm{p} \leq 0.05$ and ** $\mathrm{p} \leq 0.01$ by the Mann-Whitney test.

\section{Figure 7 Electron microscopic validation of the presence of Gq-DREADDs on neurons}

(a) DREADD molecules labeled by silver intensified gold (SIG) particles were present on the plasma membrane (white wedges) and in the cytoplasm (need to point to it with a symbol) of Layer 5 mPFC pyramidal neurons' cell body. The plasma membrane of an adjacent non-transfected pyramidal neuron with almost no Gq-DREADD-mCherryimmunoreactivity is shown on the right (red neuron). Scale bar $=500 \mathrm{~nm}$. (b) SIG, reflective of Gq-DREADD/mCherry that was present on the plasma membrane of an apical dendrite (qA). Scale bar $=1 \mu \mathrm{m}$. (c) SIG reflective of Gq-DREADD/mCherry that was present along the plasma membrane of an dendritic spine head (white asterisk) forming an excitatory synapse that showed the postsynaptic density indicated by the white arrowheads. Scale bar $=300 \mathrm{~nm} . \mathrm{N}$, nucleus; Mito, mitochondria; G, Golgi apparatus; ER, endoplasmic reticulum; T, axon terminal; qA, apical dendrite.

\section{Figure 8 Electron microscopic quantification of GABAergic innervation of mCherry+} and mCherry-negative neurons' cell bodies in Layer 5 of mPFC

(a) Schematic illustration for the quantification of GABAergic innervation. Left, a cartoon showing an mCherry+ pyramidal (Pyr) neuron labeled by silver intensified gold particles (black dots). The GABAergic terminals (dark triangles) from the interneurons (G-IN) are distinguished from the other terminals (light triangles) due to the HRP-DAB reaction product indicating the presence of GAD. The cartoon does not reflect the relative size of a neuron and terminals. Right, an EM image showing a portion of a silver intensified gold (white arrow) labeled neuron receiving inputs from GABAergic terminals (white asterisks) and other 
terminals (black asterisk). The green area covers the entire SIG-labeled neuron, the red segments indicate the GABAergic terminals aligned with the plasma membrane. The expressions for $\%$ GABA innervation and the density of GABAergic terminals are shown on the right. Scale bar $=500 \mathrm{~nm}$. (b) Comparisons of \% GABA innervation (left panel), and the GABA terminal density (right panel), between the mCherry+ and mCherry-negative neurons. from the same DREADD experimental animal. (c) Comparisons and cumulative density funcions of the GABAergic terminal inter-synaptic lengths (rightpanel), and individual GABAergic terminal lengths (left panel) of the mCherry+ $(n=69)$ and mCherry-negative $(\mathrm{n}=83)$ neurons sampled across all of the DREADD experimental animals $(\mathrm{N}=7) .(\mathrm{d})$ Correlations between $\% \mathrm{mCherry}+/ \mathrm{cFos}+$ and $\% \mathrm{GABA}$ innervation (left panel), or GABAergic terminal density (right panel), of mCherry+ and mCherry-negative neurons. Each point represents the averaged quantities from an individual DREADD experimental animal. Significant correlation is shown with straight line together with its p-value and rsquare. $* \mathrm{p} \leq 0.05, * * \mathrm{p} \leq 0.01, * * * \mathrm{p} \leq 0.001$.

\section{Fig 9 Correlations of feeding behaviors during ABA1 with the IF and EM data}

Correlations between the food intake during the first $\mathrm{ABA}$ and $\% \mathrm{mCherry}+\mathrm{cFos}+(\mathrm{a})$, $\% \mathrm{GAD}+\mathrm{cFos}+(\mathrm{b}), \% \mathrm{GABA}$ of mCherry + neurons (c) and \%GABA of mCherry-negative neurons (d).

Fig 10 Feeding behavior during ABA2 and their correlations with the IF and EM data (C) Correlations between the food intake during the second $\mathrm{ABA}$ and $\% \mathrm{mCherry}+/ \mathrm{cFos}+(\mathrm{a})$, $\% \mathrm{GAD}+/ \mathrm{cFos}+(\mathrm{b}), \% \mathrm{GABA}$ innervation of mCherry+ neurons (c) and \%GABA innervation of mCherry-negative neurons (d). Significant correlations were indicated by straight lines 
together with their p-values and r-squares. Correlation close to significance was indicated by dashed line.

Figure 11 Summary of the proposed microcircuits in mPFC that involve the DRprojecting pyramidal neurons in this study

The DR-projecting neurons (green) are distinguished from the others because of the mCherry-tagged DREADDs on their membranes (black dots). While those neurons project to DR, they may also send outputs to the Layer 2/3 pyramidal neurons and the GABAergic neurons across layers in mPFC. Those DR-projecting neurons receive greater GABAergic innervation than other Layer 5 pyramidal neurons, which could make them more resistant to the chemogenetic excitation. Pyr, pyramidal neuron; G-IN, GABAergic interneuron; $\mathrm{DRN}^{\mathrm{GABA}}$, GABAergic neurons in DR; DRN ${ }^{5-\mathrm{HT}}$, serotonergic neuron in DR. 
REFERENCES

Amat, J., Baratta, M. V., Paul, E., Bland, S. T., Watkins, L. R., \& Maier, S. F. (2005). Medial prefrontal cortex determines how stressor controllability affects behavior and dorsal raphe nucleus. Nat Neurosci, 8(3), 365-371. doi: 10.1038/nn1399

Anastasiades, P. G., \& Carter, A. G. (2021). Circuit organization of the rodent medial prefrontal cortex. Trends Neurosci, 44(7), 550-563. doi: 10.1016/j.tins.2021.03.006

Arcelus, J., Mitchell, A. J., Wales, J., \& Nielsen, S. (2011). Mortality rates in patients with anorexia nervosa and other eating disorders. A meta-analysis of 36 studies. Arch Gen Psychiatry, 68(7), 724-731. doi: 10.1001/archgenpsychiatry.2011.74

Attia, E. (2010). Anorexia nervosa: current status and future directions. Annu Rev Med, 61, 425-435. doi: 10.1146/annurev.med.050208.200745

Ball, K., \& Lee, C. (2000). Relationships between psychological stress, coping and disordered eating: A review. Psychol Health, 14(6), 1007-1035. doi: 10.1080/08870440008407364

Bendotti, C., Garattini, S., \& Samanin, R. (1986). Hyperphagia caused by muscimol injection in the nucleus raphe dorsalis of rats: its control by 5 -hydroxytryptamine in the nucleus accumbens. J Pharm Pharmacol, 38(7), 541-543. doi: 10.1111/j.2042-7158.1986.tbo4634.x

Birmingham, C. L., Su, J., Hlynsky, J. A., Goldner, E. M., \& Gao, M. (2005). The mortality rate from anorexia nervosa. Int J Eat Disord, 38(2), 143-146.

Bulik, C. M., Slof-Op't Landt, M. C., van Furth, E. F., \& Sullivan, P. F. (2007). The genetics of anorexia nervosa. Annu Rev Nutr, 27, 263-275.

Bullitt, E. (1990). Expression of c-fos-like protein as a marker for neuronal activity following noxious stimulation in the rat. J Comp Neurol, 296(4), 517-530. doi: 10.1002/cne.902960402

Capone, F., Paolucci, M., Assenza, F., Brunelli, N., Ricci, L., Fiorio, L., \& Di Lazzaro, V. (2016). Canonical cortical circuits: current evidence and theoretical implications. Neuroscience and Neuroeconomics, 5, 1-8. doi: https://doi.org/10.2147/NAN.S70816

Celada, P., Puig, M. V., Casanovas, J. M., Guillazo, G., \& Artigas, F. (2001). Control of dorsal raphe serotonergic neurons by the medial prefrontal cortex: Involvement of serotonin-1A, $\operatorname{GABA}(\mathrm{A})$, and glutamate receptors. J Neurosci, 21(24), 9917-9929.

Chen, Y. W., Wable, G. S., Chowdhury, T. G., \& Aoki, C. (2016). Enlargement of Axo-Somatic Contacts Formed by GAD-Immunoreactive Axon Terminals onto Layer V Pyramidal Neurons in the Medial Prefrontal Cortex of Adolescent Female Mice Is Associated with Suppression of Food Restriction-Evoked Hyperactivity and Resilience to Activity-Based Anorexia. Cereb Cortex, 26(6), 2574-2589. doi: 10.1093/cercor/bhvo87

Chowdhury, T. G., Wable, G. S., Chen, Y. W., Tateyama, K., Yu, I., Wang, J. Y., ... Aoki, C. (2019). Voluntary Wheel Running Exercise Evoked by Food-Restriction Stress Exacerbates Weight Loss of Adolescent Female Rats But Also Promotes Resilience by Enhancing GABAergic Inhibition of Pyramidal Neurons in the Dorsal Hippocampus. Cereb Cortex, 29(10), 40354049. doi: 10.1093/cercor/bhy283

Chowdhury, T. G., Wable, G. S., Sabaliauskas, N. A., \& Aoki, C. (2013). Adolescent female C57BL/6 mice with vulnerability to activity-based anorexia exhibit weak inhibitory input onto hippocampal CA1 pyramidal cells. Neuroscience, 241, 250-267. doi: 10.1016/j.neuroscience.2013.03.020

Collaborators, G. B. o. D. S. (2013). Global, regional, and national incidence, prevalence, and years lived with disability for 301 acute and chronic diseases and injuries in 188 countries, 19902013: a systematic analysis for the Global Burden of Disease Study 2013. The Lancet, 386(9995). doi: 10.1016/S0140-6736(15)60692-4

Collison, J., \& Barnier, E. (2019). Eating disorders, body dysmorphic disorder, and body image pathology in female Australian models. Clinical Psychologist, 24, 155-165. doi: $10.1111 / \mathrm{cp} .12208$ 
Compan, V., Walsh, B. T., Kaye, W., \& Geliebter, A. (2015). How Does the Brain Implement Adaptive Decision Making to Eat? J Neurosci, 35(41), 13868-13878. doi: 10.1523/JNEUROSCl.260215.2015

Connan, F., Campbell, I. C., Katzman, M., Lightman, S. L., \& Treasure, J. (2003). A neurodevelopmental model for anorexia nervosa. Physiol Behav, 79(1), 13-24. doi: 10.1016/s0031-9384(03)00101-X

Correia, P. A., Matias, S., \& Mainen, Z. F. (2017). Stereotaxic Adeno-associated Virus Injection and Cannula Implantation in the Dorsal Raphe Nucleus of Mice. Bio Protoc, 7(18), e2549. doi: 10.21769/BioProtoc. 2549

Dahl, R. E. (2004). Adolescent brain development: a period of vulnerabilities and opportunities. Keynote address. Ann N Y Acad Sci, 1021, 1-22. doi: 10.1196/annals.1308.001

Dhruv, N. T. (2015). Rethinking canonical cortical circuits. Nat Neurosci, 18(11), 1538. doi: 10.1038/nn1115-1538

Fletcher, P. J., \& Davies, M. (1990). Dorsal raphe microinjection of 5-HT and indirect 5-HT agonists induces feeding in rats. Eur J Pharmacol, 184(2-3), 265-271. doi: 10.1016/00142999(90)90618-g

Foldi, C. J., Milton, L. K., \& Oldfield, B. J. (2017). The Role of Mesolimbic Reward Neurocircuitry in Prevention and Rescue of the Activity-Based Anorexia (ABA) Phenotype in Rats. Neuropsychopharmacology, 42(12), 2292-2300. doi: 10.1038/npp.2017.63

Fuhrmann, D., Knoll, L. J., \& Blakemore, S. J. (2015). Adolescence as a Sensitive Period of Brain Development. Trends Cogn Sci, 19(10), 558-566. doi: 10.1016/j.tics.2015.07.008

Garner, D. M., \& Garfinkel, P. E. (1980). Socio-cultural factors in the development of anorexia nervosa. Psychol Med, 10(4), 647-656. doi: 10.1017/s0033291700054945

Giedd, J. N., Blumenthal, J., Jeffries, N. O., Castellanos, F. X., Liu, H., Zijdenbos, A., . . Rapoport, J. L. (1999). Brain development during childhood and adolescence: a longitudinal MRI study. Nat Neurosci, 2(10), 861-863. doi: 10.1038/13158

Grahn, R. E., Will, M. J., Hammack, S. E., Maswood, S., McQueen, M. B., Watkins, L. R., \& Maier, S. F. (1999). Activation of serotonin-immunoreactive cells in the dorsal raphe nucleus in rats exposed to an uncontrollable stressor. Brain Res, 826(1), 35-43. doi: 10.1016/sooo68993(99)01208-1

Grice, D. E., Halmi, K. A., Fichter, M. M., Strober, M., Woodside, D. B., Treasure, J. T., . . Berrettini, W. H. (2002). Evidence for a susceptibility gene for anorexia nervosa on chromosome 1. Am J Hum Genet, 70(3), 787-792. doi: 10.1086/339250

Hall, J. F., Smith, K., Schnitzer, S. B., \& Hanford, P. V. (1953). Elevation of activity level in the rat following transition from ad libitum to restricted feeding. J Comp Physiol Psychol, 46(6), 429-433.

Han, W., Tellez, L. A., Perkins, M. H., Perez, I. O., Qu, T., Ferreira, J., . . de Araujo, I. E. (2018). A Neural Circuit for Gut-Induced Reward. Cell, 175(3), 887-888. doi: 10.1016/j.cell.2018.10.018

Hardaway, J. A., Crowley, N. A., Bulik, C. M., \& Kash, T. L. (2015). Integrated circuits and molecular components for stress and feeding: implications for eating disorders. Genes Brain Behav, 14(1), 85-97. doi: 10.1111/gbb.12185

Holland, A. J., Sicotte, N., \& Treasure, J. (1988). Anorexia nervosa: evidence for a genetic basis. J Psychosom Res, 32(6), 561-571. doi: 10.1016/0022-3999(88)90004-9

Hubel, C., Marzi, S. J., Breen, G., \& Bulik, C. M. (2019). Epigenetics in eating disorders: a systematic review. Mol Psychiatry, 24(6), 901-915. doi: 10.1038/s41380-018-0254-7

Hudson, J. I., Hiripi, E., Pope, H. G., Jr., \& Kessler, R. C. (2007). The prevalence and correlates of eating disorders in the National Comorbidity Survey Replication. Biol Psychiatry, 61(3), 348358. doi: 10.1016/j.biopsych.2006.03.040

Jankowski, M. P., \& Sesack, S. R. (2004). Prefrontal cortical projections to the rat dorsal raphe nucleus: ultrastructural features and associations with serotonin and gamma-aminobutyric acid neurons. J Comp Neurol, 468(4), 518-529. doi: 10.1002/cne.10976 
Jean, A., Laurent, L., Delaunay, S., Doly, S., Dusticier, N., Linden, D., .. Compan, V. (2017). Adaptive Control of Dorsal Raphe by $5-\mathrm{HT}_{4}$ in the Prefrontal Cortex Prevents Persistent Hypophagia following Stress. Cell Rep, 21(4), 901-909. doi: 10.1016/j.celrep.2017.10.003

Kaye, W. H., Fudge, J. L., \& Paulus, M. (2009). New insights into symptoms and neurocircuit function of anorexia nervosa. Nat Rev Neurosci, 10(8), 573-584. doi: 10.1038/nrn2682

Lee, A. T., Gee, S. M., Vogt, D., Patel, T., Rubenstein, J. L., \& Sohal, V. S. (2014). Pyramidal neurons in prefrontal cortex receive subtype-specific forms of excitation and inhibition. Neuron, 81(1), 61-68. doi: 10.1016/j.neuron.2013.10.031

Marti, O., Marti, J., \& Armario, A. (1994). Effects of chronic stress on food intake in rats: influence of stressor intensity and duration of daily exposure. Physiol Behav, 55(4), 747-753. doi: 10.1016/0031-9384(94)90055-8

Merikangas, K. R., He, J. P., Burstein, M., Swanson, S. A., Avenevoli, S., Cui, L., ... Swendsen, J. (2010). Lifetime prevalence of mental disorders in U.S. adolescents: results from the National Comorbidity Survey Replication--Adolescent Supplement (NCS-A). J Am Acad Child Adolesc Psychiatry, 49(10), 980-989. doi: 10.1016/j.jaac.2010.05.017

Nectow, A. R., Schneeberger, M., Zhang, H., Field, B. C., Renier, N., Azevedo, E., ... Friedman, J. M. (2017). Identification of a Brainstem Circuit Controlling Feeding. Cell, 170(3), 429-442 e411. doi: 10.1016/j.cell.2017.06.045

Nichols, I. S., Jones, M. I., Okere, C., Ananaba, G., Bush, B., Gray, C., ... Paul, K. (2017). Nitrergic neurons of the dorsal raphe nucleus encode information about stress duration. PLoS One, 12(11), e0187071. doi: 10.1371/journal.pone.0187071

Nishitani, N., Nagayasu, K., Asaoka, N., Yamashiro, M., Andoh, C., Nagai, Y., ... Kaneko, S. (2019). Manipulation of dorsal raphe serotonergic neurons modulates active coping to inescapable stress and anxiety-related behaviors in mice and rats. Neuropsychopharmacology, 44(4), 721732. doi: 10.1038/s41386-018-0254-y

Petrovich, G. D., Holland, P. C., \& Gallagher, M. (2005). Amygdalar and prefrontal pathways to the lateral hypothalamus are activated by a learned cue that stimulates eating. J Neurosci, 25(36), 8295-8302. doi: 10.1523/JNEUROSCl.2480-05.2005

Polivy, J., \& Herman, C. P. (2002). Causes of eating disorders. Annu Rev Psychol, 53, 187-213. doi: 10.1146/annurev.psych.53.100901.135103

Pollano, A., Trujillo, V., \& Suarez, M. M. (2018). How does early maternal separation and chronic stress in adult rats affect the immunoreactivity of serotonergic neurons within the dorsal raphe nucleus? Stress, 21(1), 59-68. doi: 10.1080/10253890.2017.1401062

Reiner, A., Jiao, Y., Del Mar, N., Laverghetta, A. V., \& Lei, W. L. (2003). Differential morphology of pyramidal tract-type and intratelencephalically projecting-type corticostriatal neurons and their intrastriatal terminals in rats. J Comp Neurol, 457(4), 420-440. doi: 10.1002/cne.10541

Roux, P., Blanchard, J. M., Fernandez, A., Lamb, N., Jeanteur, P., \& Piechaczyk, M. (1990). Nuclear localization of c-Fos, but not v-Fos proteins, is controlled by extracellular signals. Cell, 63(2), 341-351. doi: 10.1016/0092-8674(90)90167-d

Santiago, A. N., Makowicz, E. A., Du, M., \& Aoki, C. (2021). Food Restriction Engages Prefrontal Corticostriatal Cells and Local Microcircuitry to Drive the Decision to Run versus Conserve Energy. Cereb Cortex, 31(6), 2868-2885. doi: 10.1093/cercor/bhaa394

Shepherd, G. M. (2013). Corticostriatal connectivity and its role in disease. Nat Rev Neurosci, 14(4), 278-291. doi: 10.1038/nrn3469

Smink, F. R., van Hoeken, D., Oldehinkel, A. J., \& Hoek, H. W. (2014). Prevalence and severity of DSM-5 eating disorders in a community cohort of adolescents. Int J Eat Disord, 47(6), 610619. doi: 10.1002/eat.22316

Strober, M., Freeman, R., Lampert, C., Diamond, J., \& Kaye, W. (2000). Controlled family study of anorexia nervosa and bulimia nervosa: evidence of shared liability and transmission of partial syndromes. Am J Psychiatry, 157(3), 393-401. doi: 10.1176/appi.ajp.157.3.393 
Sun, Q., Li, X., Ren, M., Zhao, M., Zhong, Q., Ren, Y., ... Luo, Q. (2019). A whole-brain map of longrange inputs to GABAergic interneurons in the mouse medial prefrontal cortex. Nat Neurosci, 22(8), 1357-1370. doi: 10.1038/541593-019-0429-9

Takase, L. F., \& Nogueira, M. I. (2008). Patterns of fos activation in rat raphe nuclei during feeding behavior. Brain Res, 1200, 10-18. doi: 10.1016/j.brainres.2008.01.036

Tervo, D. G., Hwang, B. Y., Viswanathan, S., Gaj, T., Lavzin, M., Ritola, K. D., .. Karpova, A. Y. (2016). A Designer AAV Variant Permits Efficient Retrograde Access to Projection Neurons. Neuron, 92(2), 372-382. doi: 10.1016/j.neuron.2016.09.021

Thompson, J. K., \& Stice, R. (2001). Thin-ideal internalization: Mounting evidence for a new risk factor for body-image disturbance and eatng pathology. Current Directions in Psychological Science, 10(5).

Timko, C. A., Defilipp, L., \& Dakanalis, A. (2019). Sex Differences in Adolescent Anorexia and Bulimia Nervosa: Beyond the Signs and Symptoms. Curr Psychiatry Rep, 21(1), 1. doi: 10.1007/s11920-019-0988-1

Wable, G. S., Chen, Y. W., Rashid, S., \& Aoki, C. (2015). Exogenous progesterone exacerbates running response of adolescent female mice to repeated food restriction stress by changing alpha4-GABAA receptor activity of hippocampal pyramidal cells. Neuroscience, 310, 322341. doi: 10.1016/j.neuroscience.2015.09.006

Warden, M. R., Selimbeyoglu, A., Mirzabekov, J. J., Lo, M., Thompson, K. R., Kim, S. Y., . . Deisseroth, K. (2012). A prefrontal cortex-brainstem neuronal projection that controls response to behavioural challenge. Nature, 492(7429), 428-432. doi: 10.1038/nature11617

Weissbourd, B., Ren, J., DeLoach, K. E., Guenthner, C. J., Miyamichi, K., \& Luo, L. (2014). Presynaptic partners of dorsal raphe serotonergic and GABAergic neurons. Neuron, 83(3), 645-662. doi: 10.1016/j.neuron.2014.06.024

White, E. L. (1989). Cortical circuits: synaptic organization of the cerebral cortex - structure, function, and theory: Birkhouser.

Wouterlood, F. G., \& Jorritsma-Byham, B. (1993). The anterograde neuroanatomical tracer biotinylated dextran-amine: comparison with the tracer Phaseolus vulgaris-leucoagglutinin in preparations for electron microscopy. J Neurosci Methods, 48(1-2), 75-87. doi: 10.1016/s0165-0270(05)80009-3 